\title{
Asymptotic Estimates of Hierarchical Modeling
}

\author{
Douglas N. Arnold* \\ Institute for Mathematics and its Applications, University of Minnesota, \\ Minneapolis, MN 55455, USA
}

Alexandre L. Madureira ${ }^{\dagger}$

Laboratório Nacional de Computação Científica, Petrópolis, RJ 25651-070, Brazil

\begin{abstract}
In this paper we propose a way to analyze certain classes of dimension reduction models for elliptic problems in thin domains. We develop asymptotic expansions for the exact and model solutions, having the thickness as small parameter. The modeling error is then estimated by comparing the respective expansions, and the upper bounds obtained make clear the influence of the order of the model and the thickness on the convergence rates. The techniques developed here allows for estimates in several norms and semi-norms, and also interior estimates (which disregards boundary layers).
\end{abstract}

\section{Introduction}

Much investigation has been done in the recent and not so recent past to take advantage of the small thickness to solve or approximate elliptic problems in thin domains. Indeed it is tempting to use dimension reduction, i.e., to pose and solve a modified problem in a region with one less dimension and then extend the reduced solution to the more general domain. It is reasonable to expect that the new problem will be simpler than the original one, but it is not easy to predict how far apart are the two solutions. In this paper we analyze the approximation properties of some classes of models for elliptic problems in thin domains, not only as the thickness of the domain goes to zero, but also as the "degree" of the models increases, in a sense that we will make clear.

We assume that the thin domain is a three-dimensional plate of the form $P^{\varepsilon}=$ $\Omega \times(-\varepsilon, \varepsilon)$, where $\Omega$ is a two-dimensional smoothly bounded region and $\varepsilon<1$ is a small positive quantity. For simplicity, we analyze the Poisson problem with vanishing Dirichlet boundary condition on the lateral boundary $\partial \Omega \times(-\varepsilon, \varepsilon)$, despite the fact that other equations and conditions are also of interest. Let $\partial P_{L}^{\varepsilon}=\partial \Omega \times$ $(-\varepsilon, \varepsilon)$ be the lateral boundary of the plate and $\partial P_{ \pm}^{\varepsilon}=\Omega \times\{-\varepsilon, \varepsilon\}$ its top and

*E-mail: arnold@ima.umn.edu

${ }^{\dagger}$ E-mail: alm@lncc.br 
bottom. We define then $u^{\varepsilon} \in H^{1}\left(P^{\varepsilon}\right)$ as the weak solution of

$$
\begin{array}{lc}
-\Delta u^{\varepsilon}=f^{\varepsilon} & \text { in } P^{\varepsilon}, \\
\frac{\partial u^{\varepsilon}}{\partial n}=g^{\varepsilon} & \text { on } \partial P_{ \pm}^{\varepsilon}, \\
u^{\varepsilon}=0 & \text { on } \partial P_{L}^{\varepsilon},
\end{array}
$$

where $f^{\varepsilon}: P^{\varepsilon} \rightarrow \mathbb{R}$ and $g^{\varepsilon}: \partial P_{ \pm}^{\varepsilon} \rightarrow \mathbb{R}$. In general, the solution of (1.1) will depend on $\varepsilon$ in a nontrivial way. In fact the above problem is a singularly perturbed one, and as $\varepsilon$ goes to zero it "loses" ellipticity. This results in the onset of boundary layers, as we make clear below.

Projecting the exact solution of (1.1) into the space of functions with polynomial dependence in the transverse direction results in a whole hierarchy of models that approximate the original problem with increasing accuracy as the semi-discrete space gets richer, and maintain the lower dimensional character. For symmetric elliptic problems, one possibility is to use a Ritz projection, ${ }^{21}$ deriving the minimum energy models. ${ }^{20,3,4,14}$

Characterizing the solution of (1.1) as the minimizer of the associated energy functional, i.e.,

$$
u^{\varepsilon}=\underset{v \in V\left(P^{\varepsilon}\right)}{\arg \min } \mathcal{J}(v), \text { where } \mathcal{J}(v)=\frac{1}{2} \int_{P^{\varepsilon}}|\underline{\nabla} v|^{2} d \underline{x}-\int_{P^{\varepsilon}} f^{\varepsilon} v d \underline{x}-\int_{\partial P_{ \pm}^{\varepsilon}} g^{\varepsilon} v d \underset{\sim}{\sim},
$$

and $V\left(P^{\varepsilon}\right)=\left\{v \in H^{1}\left(P^{\varepsilon}\right): v=0\right.$ on $\left.\partial P_{L}^{\varepsilon}\right\}$, we aim to find a "good" approximation for $u^{\varepsilon}$ searching for

$$
u^{\varepsilon}(p)=\underset{v \in \dot{H}^{1}\left(\Omega ; \mathbb{P}_{p}(-\varepsilon, \varepsilon)\right)}{\arg \min } \mathcal{J}(v),
$$

where $\stackrel{\circ}{H}^{1}\left(\Omega ; \mathbb{P}_{p}(-\varepsilon, \varepsilon)\right)$ is the space of polynomials of degree $p$ in $(-\varepsilon, \varepsilon)$ with coefficients in $\stackrel{H}{H}^{1}(\Omega)$. It immediately follows from its definition that $u^{\varepsilon}(p)$ is the Ritz projection of $u^{\varepsilon}$ into $\stackrel{\circ}{H}^{1}\left(\Omega ; \mathbb{P}_{p}(-\varepsilon, \varepsilon)\right)$, and (1.2) characterizes a minimum energy model. Observe that using higher polynomial degrees, i.e., higher order models, we obtain a hierarchy of models that furnish increasingly better solutions.

As an example, we write the model explicitly for $p=1$. Rewriting (1.2) in variational form, it is not hard to check that if $u^{\varepsilon}(1)\left(\underline{x}^{\varepsilon}\right)=\omega_{0}\left(\underset{\sim}{x^{\varepsilon}}\right)+\omega_{1}\left({\underset{\sim}{x}}^{\varepsilon}\right) x_{3}^{\varepsilon}$, then

$$
\begin{gathered}
-\Delta_{2 D} \omega_{0}=f^{0}+\varepsilon^{-1} g^{0}, \quad-\frac{\varepsilon^{2}}{3} \Delta_{2 D} \omega_{1}+\omega_{1}=f^{1}+g^{1} \quad \text { in } \Omega, \\
\omega_{0}=\omega_{1}=0 \quad \text { on } \partial \Omega,
\end{gathered}
$$

where $\Delta_{2 D}=\partial_{11}+\partial_{22}$ and

$$
\begin{aligned}
& f^{0}\left(x_{\sim}^{\varepsilon}\right)=\frac{1}{2 \varepsilon} \int_{-\varepsilon}^{\varepsilon} f^{\varepsilon}\left(\underset{\sim}{x^{\varepsilon}}, x_{3}^{\varepsilon}\right) d x_{3}^{\varepsilon}, \quad f^{1}\left(\underset{\sim}{x^{\varepsilon}}\right)=\frac{1}{2 \varepsilon} \int_{-\varepsilon}^{\varepsilon} f^{\varepsilon}\left({\underset{\sim}{\sim}}^{\varepsilon}, x_{3}^{\varepsilon}\right) x_{3}^{\varepsilon} d x_{3}^{\varepsilon}, \\
& g^{0}\left(x_{\sim}^{\varepsilon}\right)=\frac{1}{2}\left[g^{\varepsilon}\left(\underset{\sim}{x^{\varepsilon}}, \varepsilon\right)+g^{\varepsilon}\left({\underset{\sim}{x}}^{\varepsilon},-\varepsilon\right)\right], \quad g^{1}\left(x_{\sim}^{\varepsilon}\right)=\frac{1}{2}\left[g^{\varepsilon}\left(\underset{\sim}{x^{\varepsilon}}, \varepsilon\right)-g^{\varepsilon}\left({\underset{\sim}{\varepsilon}}^{\varepsilon},-\varepsilon\right)\right] .
\end{aligned}
$$


Note that the two differential equations in (1.3) are independent of each other. We can express in a unique way any function defined on $P^{\varepsilon}$ as a sum of its even and odd parts with respect to $x_{3}^{\varepsilon}$. The even parts of $f^{\varepsilon}, g^{\varepsilon}$ appear only in the equation for $\omega_{0}$, and the respective odd parts show up in the equation for $\omega_{1}$. Also, the equation determining $\omega_{1}$ is singularly perturbed, but this is not the case for the equation determining $\omega_{0}$. If higher order methods were used, we would have two independent singularly perturbed systems of equations, corresponding to the even and odd parts of $u^{\varepsilon}(p)$. A similar splitting also occurs for the linearly elastic isotropic and homogeneous plate, where the equations decouple into two independent problems corresponding to bending and stretching of the plate.

The natural question of how close $u^{\varepsilon}(p)$ is to $u^{\varepsilon}$ is not easy to answer due to the complex influence of $\varepsilon$ in both the original and model solutions. Several authors investigated various aspects of this and other related problems. For a review of the literature, see the Ph.D. thesis of Madureira. ${ }^{15}$ It is worth mentioning nevertheless the work of of Vogelius and Babuška. In a series of three remarkable papers, ${ }^{21,22,23}$ they investigated various aspects of minimum energy methods for scalar elliptic homogeneous problems in a $n$-dimensional plate, with Neumann boundary condition on the top and bottom of the domain. They started by showing how to optimally choose the semi-discrete subspace that characterizes each model. This space depends only on the coefficients of the differential equation, and a truncated outer asymptotic expansion (i.e., ignoring boundary layer terms) of the exact solution belongs to it. Then they estimated the rate of convergence of the model solution (with respect to the thickness, in the energy norm). To do this they assumed that the volume loads vanished and that the surface loading was such that boundary layer effects were of higher order than the first truncated term of the outer expansion. They then estimated the difference between the exact solution and the truncated expansion. As this quantity is certainly bigger than the error of the minimum energy model in the energy norm, they then obtained an upper bound for the modeling error. This procedure was extended by Miara ${ }^{17}$ to linearly elastic plates, again with strong restrictions on the volume and surface loads. In this case the optimal subspace might depend on the data, a clear disadvantage. Recent work by Ovaskainen and Pitkäranta ${ }^{19}$ used similar ideas to obtain more refined estimates for minimum energy methods applied to a thin linearly elastic strip under traction. Some limitations of this approach are that it is not clear how to extend it to models that are not energy minimizers, nor how to obtain sharp estimates in norms other than energy or in the interior of the domain.

Our approach differs significantly from the aforementioned ones. We estimate the modeling error not by comparing the exact and model solutions directly, but rather by first looking at the difference between the solutions and their truncated asymptotic expansions, and then comparing the asymptotic expansions. This is possible because the projection used to define each model can be used to find terms of the asymptotic expansion of the model. This allows the comparison between corresponding terms of the expansions. Schematically, this is how it works: 


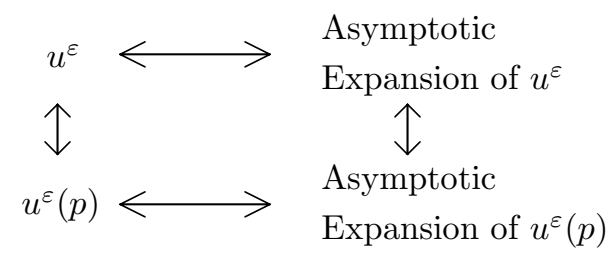

Although we also use asymptotic expansion techniques, we do not rely on the fact that our solution minimizes the potential energy. In fact our arguments work for saddle point models as well. ${ }^{15}$ In addition to the flexibility to tackle different models, we are also able to obtain sharp estimates in different norms and interior estimates.

We consider the Poisson problem as it contains the same basic characteristics and difficulties of more complex elliptic equations, but is still simple enough so that technicalities do not overshadow the main aspects of our analysis. We avoid nonetheless using specificities of the problem, and the arguments employed here extend in a natural way to the analysis of hierarchical models for linearly elastic plates. Indeed, based on the asymptotic expansions developed by Monique Dauge and her collaborators, ${ }^{8,9}$ a similar kind of study can be performed.

We now briefly introduce and explain some basic notation that we use throughout this paper. For an integer $p$ and a positive real number $a$, we define $\mathbb{P}_{p}(-a, a)$ as the space of polynomials of degree $p$ in $(-a, a)$. If $s$ is a real number and $D$ an open set, then $H^{s}(D)$ is the Sobolev space of order $s$, and $\stackrel{H}{ }^{s}(D)$ is the closure in $H^{s}(D)$ of the set of smooth functions with compact support in $D$. For $m \in \mathbb{N}$ and a separable Hilbert space $E$, we denote $H^{m}(D ; E)$ as the space of functions defined on $D$ with values in $E$ such that the $E$-norm of all partial derivatives of order less or equal to $m$ are in $L^{2}(D)$. Also, $\hat{L}^{2}(-a, a)$ is the set of square integrable functions with mean value zero in the domain $(-a, a)$, for a positive number $a$. Finally $\mathcal{D}(D)$ denotes the space of $\mathcal{C}^{\infty}$ functions in $D$ with compact support, while $\mathcal{D}^{\prime}(D)$ denotes the space of distributions.

As we have already hinted, we use one underbar for 3 -vectors and one undertilde for 2 -vectors. We can then decompose 3 -vectors as follows:

$$
\underline{u}=\left(\begin{array}{c}
u \\
\tilde{u_{3}}
\end{array}\right)
$$

We denote by lowercase $c$ a generic constant (not necessarily the same in all occurrences) which is independent not only of $\varepsilon$ and $p$, but also of $f$ and $g$, while we use uppercase $C$ when the constant may depend on $f$ and $g$, more precisely on Sobolev norms of $f$ and $g$, but not $\varepsilon$ and $p$. We denote a typical point in $P^{\varepsilon}$ by $\underline{x}^{\varepsilon}=\left(\underset{\sim}{x^{\varepsilon}}, x_{3}^{\varepsilon}\right)$, with $\underset{\sim}{x^{\varepsilon}}=\left(x_{1}^{\varepsilon}, x_{2}^{\varepsilon}\right) \in \Omega$.

Next, we outline the contents of this paper. In Section 2 we develop an asymptotic expansion for the solution of (1.1), presenting upper bounds for the difference 
between the exact solution and truncated asymptotic expansions. The following section contains the same sort of development, but this time concerning the hierarchical model solution. We present modeling error estimates in Section 4. Finally, many issues involving the boundary correctors are discussed in the appendix, including existence, uniqueness and exponential decay of solutions, approximation by polynomials, and corner singularities.

\section{Asymptotic Expansions for the Exact Solution}

We start this section by developing an asymptotic expansion for $u^{\varepsilon}$. As in Ciarlet's book, ${ }^{7}$ we define an $\varepsilon$-independent domain $P=\Omega \times(-1,1)$. A point $\underline{x}=\left(\underset{\sim}{x}, x_{3}\right)$ in $P$ is related to a point $\underline{x}^{\varepsilon}$ in $P^{\varepsilon}$ by $\underset{\sim}{x}=\underset{\sim}{x}, x_{3}=\varepsilon^{-1} x_{3}^{\varepsilon}$. We accordingly define $\partial P_{L}=\partial \Omega \times(-1,1)$, and $\partial P_{ \pm}=\Omega \widetilde{\times}\{-1,1\}$.

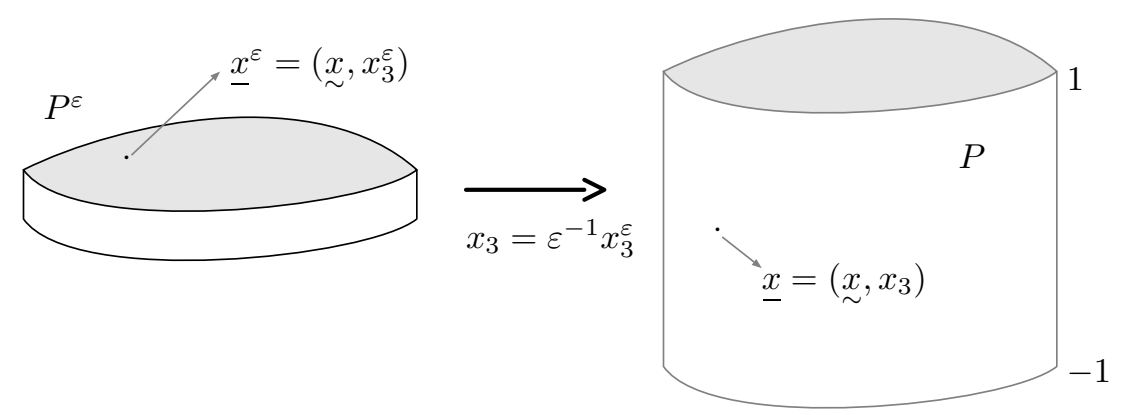

In this new domain we define $u(\varepsilon)(\underline{x})=u^{\varepsilon}\left(\underline{x}^{\varepsilon}\right), f(\underline{x})=f^{\varepsilon}\left(\underline{x}^{\varepsilon}\right)$, and $g(\underline{x})=$ $\varepsilon^{-1} g^{\varepsilon}\left(\underline{x}^{\varepsilon}\right)$. We infer from (1.1) that

$$
\begin{gathered}
\Delta_{2 D} u(\varepsilon)+\varepsilon^{-2} \partial_{33} u(\varepsilon)=-f \quad \text { in } P, \\
\frac{\partial u(\varepsilon)}{\partial n}=\varepsilon^{2} g \quad \text { on } \partial P_{ \pm}, \\
u(\varepsilon)=0 \quad \text { on } \partial P_{L} .
\end{gathered}
$$

We assume that $f, g$ are $\varepsilon$-independent, but this restriction could be relaxed, for instance by assuming that $f$ and $g$ can be represented as a power series in $\varepsilon$, plus a small remainder. ${ }^{16}$ Furthermore, how exactly $f$ and $g$ scales with respect to $\varepsilon$ is immaterial since we are considering a linear problem and the final rates of convergence are in relative norms.

Consider the asymptotic expansion

$$
u^{0}+\varepsilon^{2} u^{2}+\varepsilon^{4} u^{4}+\cdots,
$$

and formally substitute it for $u(\varepsilon)$ in (2.4). Grouping together terms with same power in $\varepsilon$ we have

$$
\begin{gathered}
\varepsilon^{-2} \partial_{33} u^{0}+\left(\Delta_{2 D} u^{0}+\partial_{33} u^{2}\right)+\varepsilon^{2}\left(\Delta_{2 D} u^{2}+\partial_{33} u^{4}\right)+\cdots=-f \\
\frac{\partial u^{0}}{\partial n}+\varepsilon^{2} \frac{\partial u^{2}}{\partial n}+\varepsilon^{4} \frac{\partial u^{4}}{\partial n}+\cdots=\varepsilon^{2} g \quad \text { on } \partial P_{ \pm}
\end{gathered}
$$


Asymptotic Estimates of Hierarchical Modeling

It is then natural to require that

$$
\begin{gathered}
\partial_{33} u^{0}=0 \\
\partial_{33} u^{2}=-f-\Delta_{2 D} u^{0} \\
\partial_{33} u^{2 k}=-\Delta_{2 D} u^{2 k-2}, \quad \text { for all } k>1
\end{gathered}
$$

along with the boundary conditions

$$
\frac{\partial u^{2 k}}{\partial n}=\delta_{k 1} g \text { on } \partial P_{ \pm}, \quad \text { for all } k \in \mathbb{N}
$$

Note that Eqs. (2.8)-(2.11) define a sequence of Neumann problems for $x_{3} \in(-1,1)$, parametrized by $\underset{\sim}{x} \in \Omega$. If the data for these problems is compatible then the solution can be written as

$$
u^{2 k}(\underline{x})=\hat{u}^{2 k}(\underline{x})+\zeta^{2 k}(\underset{\sim}{x}), \quad \text { for all } k \in \mathbb{N},
$$

where

$$
\int_{-1}^{1} \hat{u}^{2 k}\left(\underset{\sim}{x}, x_{3}\right) d x_{3}=0
$$

with $\hat{u}^{2 k}$ uniquely determined, but $\zeta^{2 k}$ an arbitrary function of $\underset{\sim}{x}$ only. As we shall see, the $\zeta^{2 k}$ will be determined using the condition of compatibility of the data for the Neumann problems. From the Dirichlet boundary condition in (2.4), it would be natural to require that $u^{2 k}=0$ on $\partial P_{L}$. This is equivalent to imposing

$$
\begin{array}{cc}
\zeta^{2 k}=0 & \text { on } \partial \Omega, \\
\hat{u}^{2 k}=0 & \text { on } \partial P_{L} .
\end{array}
$$

However, in general, only (2.14) can be imposed and (2.15) will not hold. We shall correct this discrepancy latter. Now we show that the functions $\zeta^{2 k}, \hat{u}^{2 k}$ (and so $\left.u^{2 k}\right)$ are uniquely determined from (2.8)-(2.14). In fact, (2.8) and (2.11) yields $\hat{u}^{0}=0$. From the compatibility of (2.9) and (2.11) we see that

$$
\Delta_{2 D} \zeta^{0}(\underset{\sim}{x})=-\frac{1}{2} \int_{-1}^{1} f\left(\underset{\sim}{x}, x_{3}\right) d x_{3}-\frac{1}{2}[g(\underset{\sim}{x}, 1)+g(\underset{\sim}{x},-1)]
$$

which together with (2.14), determines $\zeta^{0}$ and then, from (2.12), $u^{0}$. In view of the compatibility condition (2.16), $\hat{u}^{2}$ is fully determined by (2.9), (2.11), and (2.13). Next, the Neumann problem (2.10), (2.11) admits a solution for $k>1$ if and only if $\Delta_{2 D} \zeta^{2 k-2}=0$. But in view of (2.14), this means $\zeta^{2 k-2}=0$, for $k>1$, and then $\hat{u}^{2 k}$ is uniquely determined from $(2.10),(2.11)$. Note that $u^{0}=\zeta^{0}$ and $u^{2 k}=\hat{u}^{2 k}$ for $k \geq 1$.

Observe that $u^{0}=0$ on the lateral boundary of $P$, since $\hat{u}^{0}=0$ and so (2.15) holds for $k=0$. However, $u^{2}, u^{4}$, etc, will not in general vanish on $\partial P_{L}$ (although 
their vertical integrals do). Thus (2.5) does not give a complete asymptotic expansion of $u(\varepsilon)$ and we seek a boundary corrector $U$, which should satisfy

$$
\begin{gathered}
\Delta_{2 D} U+\varepsilon^{-2} \partial_{33} U=0 \quad \text { in } P, \\
\frac{\partial U}{\partial n}=0 \quad \text { on } \partial P_{ \pm}, \quad U \sim \varepsilon^{2} u^{2}+\varepsilon^{4} u^{4}+\cdots \quad \text { on } \partial P_{L} .
\end{gathered}
$$

To study this singular perturbation problem, we define a system of boundary-fitted horizontal coordinates. In this new system, a point close to the boundary $\partial \Omega$ has as coordinates its distance to the boundary and the arclength along the boundary to its nearest boundary point. We follow the notation of Chen. ${ }^{6}$ Suppose that $\partial \Omega$ is arclength parametrized by $\underset{\sim}{z}(\theta)=(X(\theta), Y(\theta))$. Let $\underset{\sim}{s}=\left(X^{\prime}, Y^{\prime}\right), \underset{\sim}{n}=\left(Y^{\prime},-X^{\prime}\right)$ denote the tangent and the outward normal vectors to $\widetilde{\partial} \Omega$, and define

$$
\Omega_{b}=\left\{\underset{\sim}{z}-\rho \underset{\sim}{\sim}: \underset{\sim}{z} \in \partial \Omega, 0<\rho<\rho_{0}\right\},
$$

where $\rho_{0}$ is a positive number smaller than the minimum radius of curvature of $\partial \Omega$. With $L$ denoting the arclength of $\partial \Omega$, then

$$
\underset{\sim}{x}(\rho, \theta)=\underset{\sim}{z}(\theta)-\underset{\sim}{\operatorname{co}}(\theta) .
$$

is a diffeomorphism between $\left(0, \rho_{0}\right) \times \mathbb{R} / L$ and $\Omega_{b}$. Extending $\underset{\sim}{n}$ and $\underset{\sim}{s}$ to $\Omega_{b}$ by

$$
\underset{\sim}{n}(\rho, \theta)=\underset{\sim}{n}(\theta), \quad \underset{\sim}{s}(\rho, \theta)=\underset{\sim}{s}(\theta)
$$

then, for $\alpha=1,2$ :

$$
\partial_{\alpha} \theta=\frac{s_{\alpha}}{J(\theta)}, \quad \partial_{\alpha} \rho=-n_{\alpha},
$$

where $J(\rho, \theta)=1-\rho \kappa(\theta)$, and $\kappa$ is the curvature of $\partial \Omega$. Finally, a change of coordinates yields

$$
\partial_{\alpha} f=\partial_{\theta} f \partial_{\alpha} \theta+\partial_{\rho} f \partial_{\alpha} \rho, \quad \text { for } \alpha=1,2 .
$$

The expression for the Laplacian in these new coordinates follows:

$$
\begin{aligned}
\Delta_{2 D} U=\partial_{\rho \rho} U-\frac{\kappa}{J} \partial_{\rho} U+ & \frac{1}{J^{2}} \partial_{\theta \theta} U+\frac{\rho \kappa^{\prime}}{J^{3}} \partial_{\theta} U \\
& =\partial_{\rho \rho} U+\sum_{j=0}^{\infty} \rho^{j}\left(a_{1}^{j} \partial_{\rho} U+a_{2}^{j} \partial_{\theta \theta} U+a_{3}^{j} \partial_{\theta} U\right)
\end{aligned}
$$

where we formally replace each coefficient with its respective Taylor expansion, ${ }^{1}$ and

$$
a_{1}^{j}=-[\kappa(\theta)]^{j+1}, \quad a_{2}^{j}=(j+1)[\kappa(\theta)]^{j}, \quad a_{3}^{j}=\frac{j(j+1)}{2}[\kappa(\theta)]^{j-1} \kappa^{\prime}(\theta) .
$$


Defining the new variable $\tilde{\rho}=\varepsilon^{-1} \rho$ and using the same name for functions different only up to this change of coordinates, we have from (2.19) that

$$
\Delta_{2 D} U=\varepsilon^{-2} \partial_{\tilde{\rho} \tilde{\rho}} U+\sum_{j=0}^{\infty}(\varepsilon \tilde{\rho})^{j}\left(a_{1}^{j} \varepsilon^{-1} \partial_{\tilde{\rho}} U+a_{2}^{j} \partial_{\theta \theta} U+a_{3}^{j} \partial_{\theta} U\right),
$$

Aiming to solve (2.17), we insert the asymptotic expansion

$$
U \sim \varepsilon^{2} U^{2}+\varepsilon^{3} U^{3}+\varepsilon^{4} U^{4}+\cdots,
$$

in (2.20), and collect together terms with same order of $\varepsilon$. This leads us to pose a sequence of problems in the semi-infinite strip $\Sigma=\mathbb{R}^{+} \times(-1,1)$, for $k \geq 2$ :

$$
\begin{gathered}
\left(\partial_{\tilde{\rho} \tilde{\rho}}+\partial_{33}\right) U^{k}=F_{k} \quad \text { in } \Sigma \\
\frac{\partial U^{k}}{\partial n}=0 \quad \text { on } \mathbb{R}^{+} \times\{-1,1\} \\
U^{k}\left(0, \theta, x_{3}\right)=u^{k}\left(0, \theta, x_{3}\right) \quad \text { for } x_{3} \in(-1,1),
\end{gathered}
$$

where

$$
F_{k}=\sum_{j=0}^{k-2} \tilde{\rho}^{j}\left(a_{1}^{j} \partial_{\tilde{\rho}} U^{k-j-1}+a_{2}^{j} \partial_{\theta \theta} U^{k-j-2}+a_{3}^{j} \partial_{\theta} U^{k-j-2}\right),
$$

with the convention that $u^{k}=0$ for $k$ odd and $U^{0}=U^{1}=0$. Note that the problem described by (2.22) — we show that it is well-defined further ahead - is parametrized by $\theta$, and that the geometry of $\Omega$ plays an important role through the coefficients $a_{1}^{j}, a_{2}^{j}, a_{3}^{j}$.

Combining (2.5) and (2.21) we obtain the formal asymptotic expansion

$$
u^{\varepsilon}\left(\underline{x}^{\varepsilon}\right) \sim \sum_{k=0}^{\infty} \varepsilon^{2 k} u^{2 k}\left({\underset{\sim}{x}}^{\varepsilon}, \varepsilon^{-1} x_{3}^{\varepsilon}\right)-\chi(\rho) \sum_{k=2}^{\infty} \varepsilon^{k} U^{k}\left(\varepsilon^{-1} \rho, \theta, \varepsilon^{-1} x_{3}^{\varepsilon}\right) .
$$

Here $\chi(\rho)$, is a smooth cutoff function which is identically one if $0 \leq \rho \leq \rho_{0} / 3$ and identically zero if $\rho \geq 2 \rho_{0} / 3$. (This does not turn out to be a significant source of error since $U^{k}$ decays exponentially to zero in the normal direction.)

Although our reasoning has been formal so far, we shall rigorously justify this asymptotic expansion in Theorem 2.1. Before doing that, we first study the terms entering into the expansion.

We use the following notation:

$$
\|v\|_{(m, n, P)}=\|v\|_{H^{m}\left(\Omega ; H^{n}(-1,1)\right)}, \quad\|(f, g)\|_{m, P}=\|f\|_{(m, 0, P)}+\|g\|_{H^{m}\left(\partial P_{ \pm}\right)} .
$$

In the lemma below, the bounds follow from standard regularity estimates for Eqs. (2.16), (2.9)-(2.11).

Lemma 2.1 Suppose that $f$ and $g$ are smooth functions on $P$ and $\partial P_{ \pm}$, respectively. Then the functions $u^{0}, u^{2}, \cdots$ on $P$ are uniquely determined by (2.8)-(2.14), 
and $u^{0}(\underline{x})=\zeta^{0}(\underset{\sim}{x})$ is independent of $x_{3}$. Moreover, for $m$ a nonnegative integer and $s$ a real number such that $s \geq 2$, there exists a constant $c$ independent of $f$ and $g$ such that

$$
\begin{gathered}
\left\|\zeta^{0}\right\|_{H^{m+1}(\Omega)} \leq c\|\mid(f, g)\|_{m-1, P} \\
\left\|u^{2}(\underset{\sim}{x}, \cdot)\right\|_{H^{s}(-1,1)} \leq c\left(\|f(\underset{\sim}{x}, \cdot)\|_{H^{s-2}(-1,1)}+|g(\underset{\sim}{x},-1)|+|g(\underset{\sim}{x}, 1)|\right), \\
\left\|u^{2}\right\|_{(m, s, P)} \leq c\left(\|f\|_{(m, s-2, P)}+\|g\|_{H^{m}\left(\partial P_{ \pm}\right)}\right) .
\end{gathered}
$$

The next lemma, whose proof we postpone to the appendix, guarantees the existence, uniqueness, and exponential decay of solutions for (2.22).

Lemma 2.2 Assume, for a fixed positive integer $k$, that $u^{k}$ is defined as above. Then, for each $\theta$, there exists a unique weak solution $U^{k}(\cdot, \theta, \cdot) \in H^{1}(\Sigma)$ to $(2.22)$. Also, there exist positive constants $C$ and $\alpha$ such that

$$
\int_{t}^{\infty} \int_{-1}^{1}\left(U^{k}\right)^{2}+\left(\partial_{\tilde{\rho}} U^{k}\right)^{2}+\left(\partial_{3} U^{k}\right)^{2} d x_{3} d \tilde{\rho} \leq C e^{-\alpha t}
$$

for every nonnegative real number $t$. The constant $\alpha$ may depend on $\Omega$ and $k$, but is independent of $f$ and $g$, while the constant $C$ may depend on $\Omega, k, f$, and $g$.

Although (2.23) is a formal expansion, a rigorous error estimate shows that the difference between the exact solution and a truncated asymptotic expansion is of the same order of the first term omitted in the expansion. In fact, define

$$
e_{2 N}^{\varepsilon}\left(\underline{x}^{\varepsilon}\right)=u^{\varepsilon}\left(\underline{x}^{\varepsilon}\right)-\sum_{k=0}^{N} \varepsilon^{2 k} u^{2 k}\left({\underset{\sim}{x}}^{\varepsilon}, \varepsilon^{-1} x_{3}^{\varepsilon}\right)+\chi(\rho) \sum_{k=2}^{2 N} \varepsilon^{k} U^{k}\left(\varepsilon^{-1} \rho, \theta, \varepsilon^{-1} x_{3}^{\varepsilon}\right) .
$$

In the theorem below we bound the $H^{1}\left(P^{\varepsilon}\right)$ norm of $e_{2 N}^{\varepsilon}$.

Theorem 2.1 For any positive integer $N$, there exists a constant $C$ such that the difference between the truncated asymptotic expansion and the original solution measured in the original domain is bounded as follows:

$$
\left\|e_{0}^{\varepsilon}\right\|_{H^{1}\left(P^{\varepsilon}\right)} \leq C \varepsilon^{3 / 2}, \quad\left\|e_{2 N}^{\varepsilon}\right\|_{H^{1}\left(P^{\varepsilon}\right)} \leq C \varepsilon^{2 N+1} .
$$

Since the domain $P^{\varepsilon}$ depends on $\varepsilon$, the interpretation of the convergence estimates given in Theorem 2.1 is not straightforward. The relative error is more informative in this case. For this we may use (2.26) and the triangle inequality to obtain a lower bound on the $H^{1}\left(P^{\varepsilon}\right)$ norm of the solution. The leading term of the asymptotic expansion for $u^{\varepsilon}$ is $\zeta^{0}$, unless $\zeta^{0}$ is identically zero, that is, unless the quantity

$$
-\frac{1}{2} \int_{-1}^{1} f\left({\underset{\sim}{x}}_{,}^{x} x_{3}\right) d x_{3}-\frac{1}{2}[g(\underset{\sim}{x}, 1)+g(\underset{\sim}{x},-1)]
$$

appearing on the right-hand side of (2.16) vanishes. Assuming momentarily that $\zeta^{0}$ does not vanish, then we easily conclude from (2.26) and the triangle inequality that there exists a strictly positive constant $C$ depending on $f$ and $g$, such that 
$\left\|u^{\varepsilon}\right\|_{H^{1}\left(P^{\varepsilon}\right)} \geq C \varepsilon^{1 / 2}$ for all $\varepsilon$ sufficiently small. If, on the other hand $\zeta^{0}$ vanishes, but $f$ and $g$ do not both vanish identically, then it can be seen from (2.9) and (2.11) that $u^{2}$ does not vanish. Applying the second estimate of (2.26) with $N=1$ and using the triangle inequality, we conclude $\left\|u^{\varepsilon}\right\|_{H^{1}\left(P^{\varepsilon}\right)} \geq C \varepsilon^{3 / 2}$ in this case. Thus in any case (as long as $f$ and $g$ do not both vanish identically) we have

$$
\left\|u^{\varepsilon}\right\|_{H^{1}\left(P^{\varepsilon}\right)} \geq C \nu \varepsilon^{1 / 2}, \quad \text { where } \nu= \begin{cases}1, & \zeta^{0} \neq 0 \\ \varepsilon, & \zeta^{0} \equiv 0\end{cases}
$$

Thus,

$$
\frac{\left\|e_{0}^{\varepsilon}\right\|_{H^{1}\left(P^{\varepsilon}\right)}}{\left\|u^{\varepsilon}\right\|_{H^{1}\left(P^{\varepsilon}\right)}}=O\left(\nu^{-1} \varepsilon\right), \quad \frac{\left\|e_{2 N}^{\varepsilon}\right\|_{H^{1}\left(P^{\varepsilon}\right)}}{\left\|u^{\varepsilon}\right\|_{H^{1}\left(P^{\varepsilon}\right)}}=O\left(\nu^{-1} \varepsilon^{2 N+1 / 2}\right), \quad N=1,2, \ldots
$$

It is easy to estimate the convergence in some other norms as well. For instance, in the $L^{2}\left(P^{\varepsilon}\right)$ norm, we have from the triangle inequality that

$$
\left\|e_{2 N}^{\varepsilon}\right\|_{L^{2}\left(P^{\varepsilon}\right)} \leq\left\|e_{2 N+2}^{\varepsilon}\right\|_{H^{1}\left(P^{\varepsilon}\right)}+\left\|e_{2 N+2}^{\varepsilon}-e_{2 N}^{\varepsilon}\right\|_{L^{2}\left(P^{\varepsilon}\right)} .
$$

Since

$$
\begin{aligned}
\left(e_{2 N+2}^{\varepsilon}-e_{2 N}^{\varepsilon}\right)\left(\underline{x}^{\varepsilon}\right) & =-\varepsilon^{2 N+1} U^{2 N+1}\left(\varepsilon^{-1} \rho, \theta, \varepsilon^{-1} x_{3}^{\varepsilon}\right) \\
& +\varepsilon^{2 N+2}\left[u^{2 N+2}\left(x_{\sim}^{\varepsilon}, \varepsilon^{-1} x_{3}^{\varepsilon}\right)-U^{2 N+2}\left(\varepsilon^{-1} \rho, \theta, \varepsilon^{-1} x_{3}^{\varepsilon}\right)\right],
\end{aligned}
$$

we easily conclude from a scaling argument that $\left\|e_{2 N}^{\varepsilon}\right\|_{L^{2}\left(P^{\varepsilon}\right)}=O\left(\varepsilon^{2 N+2}\right)$, for $N$ positive.

Using similar arguments, it is possible to compute interior estimates, which achieve better convergence in regions far away from the lateral boundary of the plate. The reason for the improvement in such subdomains is that the influence of the boundary layer is negligible. Table 1 presents these interior and various other error estimates. We assume that $f$ and $g$ are sufficiently smooth functions and we show only the order of the norms with respect to $\varepsilon$. "BL" stands for "Boundary Layer" and the "Relative Error" column presents the norm of $e_{2 N}^{\varepsilon}$ divided by the norm of $u^{\varepsilon}$. In parentheses are the interior estimates, when these are better than the global estimates.

The remainder of this section contains the proof of Theorem 2.1. In our demonstration, we follow the basic steps of a similar proof for an elasticity problem. ${ }^{8}$

Definition 2.1 Set

$$
u_{2 N}(\underline{x})=\sum_{k=0}^{N} \varepsilon^{2 k} u^{2 k}(\underline{x}), \quad U_{2 N}(\underline{x})=\sum_{k=2}^{2 N} \varepsilon^{k} U^{k}\left(\varepsilon^{-1} \rho, \theta, x_{3}\right) .
$$

Some results regarding the boundary layer terms are collected below. 
Table 1: Order with respect to the thickness of the exact solution, the first term of the boundary layer expansion, and the difference between the solution and a truncated asymptotic expansion in various norms.

\begin{tabular}{c|c|c|c|c}
\hline norm & $u^{\varepsilon}$ & $\mathrm{BL}$ & $e_{2 N}^{\varepsilon}, N \geq 1$ & Relative Error \\
\hline$\|\cdot\|_{L^{2}\left(P^{\varepsilon}\right)}$ & $\nu^{2} \varepsilon^{\frac{1}{2}}$ & $\varepsilon^{3}$ & $\varepsilon^{2 N+2}\left(\varepsilon^{2 N+\frac{5}{2}}\right)$ & $\nu^{-2} \varepsilon^{2 N+\frac{3}{2}}\left(\nu^{-2} \varepsilon^{2 N+2}\right)$ \\
$\left\|\partial_{\rho} \cdot\right\|_{L^{2}\left(P^{\varepsilon}\right)}$ & $\nu^{\frac{3}{2}} \varepsilon^{\frac{1}{2}}\left(\nu^{2} \varepsilon^{\frac{1}{2}}\right)$ & $\varepsilon^{2}$ & $\varepsilon^{2 N+1}\left(\varepsilon^{2 N+\frac{5}{2}}\right)$ & $\nu^{-\frac{3}{2}} \varepsilon^{2 N+\frac{1}{2}}\left(\nu^{-2} \varepsilon^{2 N+2}\right)$ \\
$\left\|\partial_{\theta} \cdot\right\|_{L^{2}\left(P^{\varepsilon}\right)}$ & $\nu^{2} \varepsilon^{\frac{1}{2}}$ & $\varepsilon^{3}$ & $\varepsilon^{2 N+2}\left(\varepsilon^{2 N+\frac{5}{2}}\right)$ & $\nu^{-2} \varepsilon^{2 N+\frac{3}{2}}\left(\nu^{-2} \varepsilon^{2 N+2}\right)$ \\
$\left\|\partial_{x_{3}^{\varepsilon}} \cdot\right\|_{L^{2}\left(P^{\varepsilon}\right)}$ & $\varepsilon^{\frac{3}{2}}$ & $\varepsilon^{2}$ & $\varepsilon^{2 N+1}\left(\varepsilon^{2 N+\frac{3}{2}}\right)$ & $\varepsilon^{2 N-\frac{1}{2}}\left(\varepsilon^{2 N}\right)$ \\
$\|\cdot\|_{H^{1}\left(P^{\varepsilon}\right)}$ & $\nu \varepsilon^{\frac{1}{2}}$ & $\varepsilon^{2}$ & $\varepsilon^{2 N+1}\left(\varepsilon^{2 N+\frac{3}{2}}\right)$ & $\nu^{-1} \varepsilon^{2 N+\frac{1}{2}}\left(\nu^{-1} \varepsilon^{2 N+1}\right)$ \\
\hline
\end{tabular}

Lemma 2.3 For any positive integer $N$, there exists positive constants $C$ and $\alpha$ such that

$$
\left\|\chi^{\prime} U_{2 N}\right\|_{L^{2}(P)}+\varepsilon\left\|\chi^{\prime} \partial_{\rho} U_{2 N}\right\|_{L^{2}(P)} \leq C \varepsilon^{5 / 2} \exp \left(-\alpha \varepsilon^{-1}\right) .
$$

Also, for all $v \in H^{1}(P)$ that vanishes on $\partial P_{L}$,

$$
\left|\int_{P} \underset{\sim}{\nabla} U_{2 N} \underset{\sim}{\nabla}(\chi v)+\varepsilon^{-2} \partial_{3} U_{2 N} \partial_{3}(v \chi) d \underline{x}\right| \leq C \varepsilon^{2 N}\|v\|_{H^{1}(P)} .
$$

Proof. The inequalities (2.29) follow from a change of coordinates, Lemma 2.2, and the definition of $\chi$. To see that (2.30) holds, first rewrite (2.19) as a finite series, using Taylor expansion with remainders. Then the result follows from the definition of $U_{2 N},(2.22)$, and Lemma $2.2 \square$.

We obtain now a rough estimate for the asymptotic expansion error.

Lemma 2.4 For any positive integer $N$, let $e_{2 N}(\underline{x})=e_{2 N}^{\varepsilon}\left(\underline{x}^{\varepsilon}\right)$. Then there exists a constant $C$ such that

$$
\left\|e_{2 N}\right\|_{H^{1}(P)} \leq C \varepsilon^{2 N}
$$

Proof. We use in this proof that $u_{2 N}$ solves the Poisson problem up to arbitrary powers of $\varepsilon$. First note that $e_{2 N}$ vanishes on $\partial P_{L}$. Hence, in view of the Poincaré's inequality,

$$
\left\|e_{2 N}\right\|_{H^{1}(P)}^{2} \leq c \int_{P}\left|\underset{\sim}{\nabla} e_{2 N}\right|^{2}+\varepsilon^{-2}\left(\partial_{3} e_{2 N}\right)^{2} d \underline{x}
$$

and we estimate next the right hand side of (2.31). Let $v \in H^{1}(P)$ such that $v=0$ on $\partial P_{L}$. If we define

$$
E(2 N, v)=\int_{P} \underset{\sim}{\nabla}\left(u(\varepsilon)-u_{2 N}\right) \underset{\sim}{\nabla} v+\varepsilon^{-2} \partial_{3}\left(u(\varepsilon)-u_{2 N}\right) \partial_{3} v d \underset{\sim}{x},
$$


then, by construction of the asymptotic expansion, we have

$$
\begin{array}{r}
E(2 N, v)=\int_{P} f v d \underset{\sim}{x}+\int_{\partial P_{ \pm}} g v d \underset{\sim}{x}-\sum_{k=0}^{N} \varepsilon^{2 k} \int_{P}\left(\underset{\sim}{\nabla} u^{2 k} \underset{\sim}{\nabla} v+\varepsilon^{-2} \partial_{3} u^{2 k} \partial_{3} v\right) d \underset{\sim}{x} \\
=-\varepsilon^{2 N} \int_{P} \underset{\sim}{\nabla} u^{2 N} \underset{\sim}{\nabla} v d \underset{\sim}{x},
\end{array}
$$

and we conclude that

$$
|E(2 N, v)| \leq C \varepsilon^{2 N}\|v\|_{H^{1}(P)}
$$

We also have

$$
\begin{array}{r}
\left|\int_{P} \underset{\sim}{\nabla}\left(\chi U_{2 N}\right) \underset{\sim}{\nabla} v-\underset{\sim}{\nabla} U_{2 N} \underset{\sim}{\nabla}(\chi v)+\varepsilon^{-2}\left[\partial_{3}\left(\chi U_{2 N}\right) \partial_{3} v-\partial_{3} U_{2 N} \partial_{3}(\chi v)\right] d x\right| \\
\leq\left(\left\|\chi^{\prime} U_{2 N}\right\|_{L^{2}(P)}+\left\|\chi^{\prime} \partial_{\rho} U_{2 N}\right\|_{L^{2}(P)}\right)\|v\|_{H^{1}(P)} .
\end{array}
$$

Hence, by Lemma 2.3

$$
\left|\int_{P}\left[\underset{\sim}{\nabla}\left(\chi U_{2 N}\right) \underset{\sim}{\nabla} v+\varepsilon^{-2} \partial_{3}\left(\chi U_{2 N}\right) \partial_{3} v\right] d \underset{\sim}{x}\right| \leq C \varepsilon^{2 N}\|v\|_{H^{1}(P)} .
$$

Making $v=e_{2 N}$ we have

$$
\begin{aligned}
& \int_{P}\left|\underset{\sim}{\nabla} e_{2 N}\right|^{2}+\varepsilon^{-2}\left(\partial_{3} e_{2 N}\right)^{2} \underset{\sim}{d x} \\
&=E\left(2 N, e_{2 N}\right)+\int_{P}\left[\underset{\sim}{\nabla}\left(\chi U_{2 N}\right) \underset{\sim}{\nabla} e_{2 N}+\varepsilon^{-2} \partial_{3}\left(\chi U_{2 N}\right) \partial_{3} e_{2 N}\right] d \underset{\sim}{x} \\
& \leq C \varepsilon^{2 N}\left\|e_{2 N}\right\|_{H^{1}(P)}
\end{aligned}
$$

from (2.32) and (2.33), and the result follows from (2.31) $\square$.

The estimate in Lemma 2.4 is not sharp. The powers of $\varepsilon$ can be shown to be $2 N+1 / 2$. We make this improvement when we consider the error on the unscaled plate $P^{\varepsilon}$.

Proof. (of Theorem 2.1) Assume first that $N$ is positive. From Lemma 2.4, we immediately obtain $\left\|e_{2 N}^{\varepsilon}\right\|_{H^{1}\left(P^{\varepsilon}\right)}=O\left(\varepsilon^{2 N-1 / 2}\right)$. This result too is not sharp. To obtain a sharp result, we use the triangle inequality:

$$
\left\|e_{2 N}^{\varepsilon}\right\|_{H^{1}\left(P^{\varepsilon}\right)} \leq\left\|e_{2 N+2}^{\varepsilon}-e_{2 N}^{\varepsilon}\right\|_{H^{1}\left(P^{\varepsilon}\right)}+O\left(\varepsilon^{2 N+3 / 2}\right),
$$

and then the result follows from (2.28) and a scaling argument. A similar argument holds for $N=0 \square$.

\section{Asymptotic Expansions for the Model Solution}

To develop an asymptotic expansion for the solution of the hierarchical models, we reason as before, but use weak equations instead of their strong form. We start 
by posing a problem for the solution of the minimum energy model in the scaled domain $P$. If we define $u(p)(\underline{x})=u^{\varepsilon}(p)\left(\underline{x}^{\varepsilon}\right)$, then

$$
\int_{P} \underset{\sim}{\nabla} u(p) \underset{\sim}{\nabla} v+\varepsilon^{-2} \partial_{3} u(p) \partial_{3} v d \underset{\sim}{\sim}=\int_{P} f v d \underset{\sim}{x}+\int_{\partial P_{ \pm}} g v d x
$$

for all $v \in \stackrel{\circ}{H}^{1}\left(\Omega ; \mathbb{P}_{p}(-1,1)\right)$. Considering the asymptotic expansion

$$
u^{0}(p)+\varepsilon^{2} u^{2}(p)+\varepsilon^{4} u^{4}(p)+\cdots,
$$

and formally substituting it for $u(p)$ in (3.34), we conclude that,

$$
\begin{gathered}
\int_{P} \partial_{3} u^{0}(p) \partial_{3} v d x=0, \\
\int_{P} \partial_{3} u^{2}(p) \partial_{3} v \underset{\sim}{d x}=\int_{P}\left(f+\Delta_{2 D} u^{0}(p)\right) v \underset{\sim}{d x}+\int_{\partial P_{ \pm}} g v d \underset{\sim}{x} \\
\int_{P} \partial_{3} u^{2 k}(p) \partial_{3} v \underset{\sim}{d x}=\int_{P} \Delta_{2 D} u^{2 k-2}(p) v \underset{\sim}{d x}, \quad \text { for } k>1,
\end{gathered}
$$

for all $v \in \stackrel{\circ}{H}^{1}\left(\Omega ; \mathbb{P}_{p}(-1,1)\right)$. Let $\hat{\mathbb{P}}_{p}(-1,1)$ be the space of polynomials of degree $p$ in $(-1,1)$ with zero average. Repeating the arguments of the expansion for the exact solution, we set $u^{0}(p)(\underline{x})=\zeta^{0}(\underset{\sim}{x})$ and $\left.u^{2}(p) \underset{\sim}{x}, \cdot\right)$ as the Galerkin projection of $u^{2}(\underset{\sim}{x}, \cdot)$ into $\hat{\mathbb{P}}_{p}(-1,1)$ for almost every $\underset{\sim}{x} \in \Omega$, i.e.,

$$
\begin{aligned}
\int_{-1}^{1} \partial_{3} u^{2}(p)\left(\underset{\sim}{x}, x_{3}\right) \partial_{3} v\left(x_{3}\right) d x_{3}=\int_{-1}^{1}\left[f\left(\underset{\sim}{x}, x_{3}\right)+\Delta_{2 D} \zeta^{0}(\underset{\sim}{x})\right] v\left(x_{3}\right) d x_{3} \\
+g(\underset{\sim}{x},-1) v(-1)+g(\underset{\sim}{x}, 1) v(1), \quad \text { for all } v \in \hat{\mathbb{P}}_{p}(-1,1) .
\end{aligned}
$$

For any integer $k \geq 2$, we define $\left.u^{2 k}(p) \underset{\sim}{x}, \cdot\right) \in \hat{\mathbb{P}}_{p}(-1,1)$ by

$$
\left.\int_{-1}^{1} \partial_{3} u^{2 k}(p)\left(\underset{\sim}{x}, x_{3}\right) \partial_{3} v\left(x_{3}\right) d x_{3}=\int_{-1}^{1} \Delta_{2 D} u^{2 k-2}(p) \underset{\sim}{x}, x_{3}\right) v\left(x_{3}\right) d x_{3},
$$

for all $v \in \hat{\mathbb{P}}_{p}(-1,1)$, and for almost every $\underset{\sim}{x} \in \Omega$.

The ansatz (3.35) does not satisfy the Dirichlet boundary conditions at $\partial P_{L}$ and we use then boundary correctors $U^{k}(p)$. These functions are polynomial in the transverse direction, and are defined in the semi-infinite strip $\Sigma$. We need to define the spaces

$$
\begin{gathered}
V(\Sigma, p)=\left\{v \in \mathcal{D}^{\prime}\left(\mathbb{R}^{+} ; \mathbb{P}_{p}(-1,1)\right):\|\underset{\sim}{\nabla} v\|_{L^{2}(\Sigma)}+\|v(0, \cdot)\|_{L^{2}(-1,1)}<\infty\right\}, \\
V_{0}(\Sigma, p)=\{v \in V(\Sigma, p): v(0, \cdot)=0\} .
\end{gathered}
$$


For any positive integer $k$, let $U^{k}(p) \in V(\Sigma, p)$ be the solutions of

$$
\begin{gathered}
\int_{\Sigma} \partial_{\tilde{\rho}} U^{k}(p) \partial_{\tilde{\rho}} v+\partial_{3} U^{k}(p) \partial_{3} v d \tilde{\rho} d x_{3}=\int_{\Sigma} F_{k}(p) v d \tilde{\rho} d x_{3} \quad \text { for all } v \in V_{0}(\Sigma, p), \\
U^{k}(p)\left(0, \theta, x_{3}\right)=u^{k}(p)\left(0, \theta, x_{3}\right) \quad \text { for all } x_{3} \in(-1,1), \\
F_{k}(p)=\sum_{j=0}^{k-1} \tilde{\rho}^{j}\left(a_{1}^{j} \partial_{\tilde{\rho}} U^{k-j-1}(p)+a_{2}^{j} \partial_{\theta \theta} U^{k-j-2}(p)+a_{3}^{j} \partial_{\theta} U^{k-j-2}(p)\right),
\end{gathered}
$$

where $u^{k}=0$ for $k$ odd and $U^{0}(p)=U^{1}(p)=0$.

A result analogous to Lemma 2.2 holds for $U^{k}(p)$ as well, guaranteeing existence, uniqueness and exponential decay, with the same decaying rate. ${ }^{15}$ This implies in particular that there exist constants $C$ and $\alpha$ such that

$$
\int_{\Sigma}\left[\chi^{\prime}(\varepsilon \tilde{\rho}) U^{2}(p)\right]^{2} d \tilde{\rho} d x_{3} \leq C \exp \left(-\alpha \varepsilon^{-1}\right)
$$

The above inequality will be of use further on. Similarly to (2.23), we have that

$$
u^{\varepsilon}(p)\left(\underline{x}^{\varepsilon}\right) \sim \zeta^{0}\left(\underline{\sim}^{\varepsilon}\right)+\sum_{k=1}^{\infty} \varepsilon^{2 k} u^{2 k}(p)\left({\underset{\sim}{x}}^{\varepsilon}, \varepsilon^{-1} x_{3}^{\varepsilon}\right)-\chi(\rho) \sum_{k=2}^{\infty} \varepsilon^{k} U^{k}(p)\left(\varepsilon^{-1} \rho, \theta, \varepsilon^{-1} x_{3}^{\varepsilon}\right),
$$

where $\zeta^{0}$ solves (2.16).

We present next an estimate, in the $H^{1}\left(P^{\varepsilon}\right)$ norm, of $u^{\varepsilon}(p)$ minus its truncated asymptotic expansion. Since the proofs of the previous section work here with minor modifications, we refrain from repeating them. We would like to remark that this result gives a bound that is uniform in $p$, and that the bound is the same (up to a constant) as in Theorem 2.1.

Theorem 3.2 For any positive integer $N$, let

$$
\begin{aligned}
e_{2 N}^{\varepsilon}(p)\left(\underline{x}^{\varepsilon}\right)=u^{\varepsilon}(p)\left(\underline{x}^{\varepsilon}\right)-\sum_{k=0}^{N} \varepsilon^{2 k} u^{2 k}(p)\left(\underline{\sim}_{\sim}^{\varepsilon}, \varepsilon^{-1} x_{3}^{\varepsilon}\right)+ & \\
& \chi(\rho) \sum_{k=2}^{2 N} \varepsilon^{k} U^{k}(p)\left(\varepsilon^{-1} \rho, \theta, \varepsilon^{-1} x_{3}^{\varepsilon}\right) .
\end{aligned}
$$

Then there exists a constant $C$ such that $\left\|e_{2 N}^{\varepsilon}(p)\right\|_{H^{1}\left(P^{\varepsilon}\right)} \leq C \varepsilon^{2 N+1}$, for all $p \in \mathbb{N}$.

\section{Estimates for the modeling error}

In this section, we estimate the modeling error. As we mentioned before, this is done by comparing the asymptotic expansions of the exact and model solution. A key point is to estimate the difference between terms of the respective expansions. We need the following definitions. 
Definition 4.2 For a nonnegative real number s, let

$$
\begin{aligned}
& a_{s}=\|f\|_{L^{2}\left(\Omega ; H^{s}(-1,1)\right)}+\|g\|_{L^{2}\left(\partial P_{ \pm}\right)}, \quad a_{s}^{1}=\|f\|_{H^{1}\left(\Omega ; H^{s}(-1,1)\right)}+\|g\|_{H^{1}\left(\partial P_{ \pm}\right)}, \\
& a_{s}^{b}=\left(\int_{\partial \Omega}\|f(\underset{\sim}{x}, \cdot)\|_{H^{s}(-1,1)}^{2}+|g(\underset{\sim}{x},-1)|^{2}+|g(\underset{\sim}{x}, 1)|^{2} d \underset{\sim}{x}\right)^{1 / 2} .
\end{aligned}
$$

The comparison between $u^{2}(p)$ and $u^{2}$ is straightforward since the former is a Galerkin projection of the latter. Indeed, let $\hat{\pi}_{p}^{1}$ be the orthogonal projection operator from $H^{1}(-1,1) \cap \hat{L}^{2}(-1,1)$ to $\hat{\mathbb{P}}_{p}(-1,1)$, with respect to the inner product that induces the norm $|\cdot|_{H^{1}(-1,1)}$. The next classical result, ${ }^{5}$ estimates the projection error.

Lemma 4.5 For any nonnegative real number $s$, there exists a constant $C$ such that if $0 \leq r \leq 1 \leq s$, then

$$
\left\|\phi-\hat{\pi}_{p}^{1} \phi\right\|_{H^{r}(-1,1)} \leq C p^{r-s}\|\phi\|_{H^{s}(-1,1)} \quad \text { for } \phi \in H^{s}(-1,1) \cap \hat{L}^{2}(-1,1) .
$$

From (2.9), (2.11)-(2.14), and (3.39), we gather that $u^{2}(p)=\hat{\pi}_{p}^{1} u^{2}$, for all $\underset{\sim}{x} \in \Omega$. From Lemmas 4.5, and 2.1, we conclude the following result.

Lemma 4.6 For any nonnegative real number $s$, there exists a constant $c$ independent of $\varepsilon, p, f$, and $g$, such that

$$
\begin{gathered}
\left\|u^{2}-u^{2}(p)\right\|_{L^{2}(P)} \leq c p^{-2-s} a_{s}, \\
\left\|\underset{\sim}{\nabla} u^{2}-\underset{\sim}{\nabla} u^{2}(p)\right\|_{L^{2}(P)} \leq c p^{-2-s} a_{s}^{1}, \\
\left\|\partial_{x_{3}} u^{2}-\partial_{x_{3}} u^{2}(p)\right\|_{L^{2}(P)} \leq c p^{-1-s} a_{s} .
\end{gathered}
$$

Bounding the difference $U^{2}-U^{2}(p)$ is harder due to the presence of corner singularities. Since both $U^{2}$ and $U^{2}(p)$ are originally defined in the semi-infinite strip $\Sigma$, it is natural to investigate the approximation properties in this domain, and such is done in the appendix. We apply these approximation results to estimate the difference between boundary correctors in $P^{\varepsilon}$.

Definition 4.3 Let $\underset{\sim}{x} \in \partial \Omega$, and let $s$ be a nonnegative real number. Let

$$
N(s)=\max \{n \in \mathbb{Z}: 2 n<s\} .
$$

If $\sup _{x_{3} \in\{-1,1\}}\left|g\left(\underset{\sim}{x}, x_{3}\right)\right| \neq 0$, set $m=1$. If $|g(\underset{\sim}{x},-1)|=|g(\underset{\sim}{x}, 1)|=0$ and

$$
\sup _{x_{3} \in\{-1,1\}} \sum_{j=2}^{N(s+5 / 2)}\left|\partial_{3}^{2 j-3} f\left(\underset{\sim}{x}, x_{3}\right)\right| \neq 0,
$$

let $m$ be the minimum integer in $\{2, \ldots, N(s+5 / 2)\}$ such that

$$
\sup _{x_{3} \in\{-1,1\}}\left|\partial_{3}^{2 m-3} f\left(\underset{\sim}{x}, x_{3}\right)\right| \neq 0 .
$$

We define in both cases $\mu(\underset{\sim}{x}, s, \delta)=\min \{4 m-2-\delta, s+3 / 2\}$. If $|g(\underset{\sim}{x},-1)|=$ $|g(\underset{\sim}{x}, 1)|=0$ and $(4.45)$ does not hold, then define $\mu \underset{\sim}{x}, s, \delta)=s+3 / 2$. Finally, set

$$
\bar{\mu}(s, \delta)=\inf _{\sim} \in \partial \Omega(\underset{\sim}{x}, s, \delta) .
$$


Remark 4.1 Four our purposes, the minimum value that $\bar{\mu}(s, \delta)$ can assume is $2-\delta$, since we will always impose $s>3 / 2$.

We postpone the proof of the next lemma to the Appendix B.

Lemma 4.7 Let $Z\left(x^{\varepsilon}\right)=\chi(\rho)\left[U^{2}-U^{2}(p)\right]\left(\varepsilon^{-1} \rho, \theta, \varepsilon^{-1} x_{3}^{\varepsilon}\right)$. For any nonnegative real number $s$ such that $s+1 / 2$ is not an even integer, and for any arbitrarily small $\delta>0$, there exists a constant $c$ independent of $\varepsilon, p, f$, and $g$, such that

$$
\left\|\partial_{\rho} Z\right\|_{L^{2}\left(P^{\varepsilon}\right)}+\left\|\partial_{x_{3}^{\varepsilon}} Z\right\|_{L^{2}\left(P^{\varepsilon}\right)} \leq c p^{-\bar{\mu}(s, \delta)} a_{s}^{b} .
$$

Finally, we present the convergence results for the hierarchical models. Let $P_{0}^{\varepsilon}=$ $\Omega_{0} \times(-\varepsilon, \varepsilon)$, here $\Omega_{0}$ is an open domain such that $\bar{\Omega}_{0} \subset \Omega$. This new domain is useful to obtain interior estimates.

Theorem 4.3 For any nonnegative real numbers $s$ and $s^{*}$ such that $s^{*}+1 / 2$ is not an even integer, and for any arbitrarily small $\delta>0$, there exist constants $c$ and $C$ independent of $\varepsilon$ and $p$, with $c$ also independent of $f$ and $g$, such that the error between $u^{\varepsilon}$ and its approximation $u^{\varepsilon}(p)$ is bounded as

$$
\begin{gathered}
\left\|u^{\varepsilon}-u^{\varepsilon}(p)\right\|_{L^{2}\left(P^{\varepsilon}\right)} \leq c \varepsilon^{5 / 2} p^{-2-s} a_{s}+C \varepsilon^{3}, \\
\left\|\partial_{\rho}\left[u^{\varepsilon}-u^{\varepsilon}(p)\right]\right\|_{L^{2}\left(P^{\varepsilon}\right)} \leq c \varepsilon^{2} p^{-\bar{\mu}\left(s^{*}, \delta\right)} a_{s^{*}}^{b}+C \varepsilon^{5 / 2}, \\
\left\|\partial_{\theta}\left[u^{\varepsilon}-u^{\varepsilon}(p)\right]\right\|_{L^{2}\left(P^{\varepsilon}\right)} \leq c \varepsilon^{5 / 2} p^{-2-s} a_{s}^{1}+C \varepsilon^{3}, \\
\left\|\underset{\sim}{\nabla} u^{\varepsilon}-\underset{\sim}{\nabla} u^{\varepsilon}(p)\right\|_{L^{2}\left(P_{0}^{\varepsilon}\right)} \leq c \varepsilon^{5 / 2} p^{-2-s} a_{s}^{1}+C \varepsilon^{9 / 2}, \\
\left\|\partial_{x_{3}^{\varepsilon}} u^{\varepsilon}-\partial_{x_{3}^{\varepsilon}} u^{\varepsilon}(p)\right\|_{L^{2}\left(P^{\varepsilon}\right)} \leq c \varepsilon^{3 / 2} p^{-1-s} a_{s}+C \varepsilon^{2}, \\
\left\|u^{\varepsilon}-u^{\varepsilon}(p)\right\|_{H^{1}\left(P^{\varepsilon}\right)} \leq c \varepsilon^{3 / 2} p^{-1-s} a_{s}+C \varepsilon^{2} .
\end{gathered}
$$

Moreover, if $f \equiv 0$, then $\left\|u^{\varepsilon}-u^{\varepsilon}(p)\right\|_{H^{1}\left(P^{\varepsilon}\right)} \leq c \varepsilon^{2} p^{-\bar{\mu}\left(s^{*}, \delta\right)} a_{s^{*}}^{b}+C \varepsilon^{5 / 2}$.

Proof. We prove the second estimate. Using the triangle inequality, the following holds:

$$
\begin{aligned}
\left\|\partial_{\rho}\left[u^{\varepsilon}-u^{\varepsilon}(p)\right]\right\|_{L^{2}\left(P^{\varepsilon}\right)} \leq & \left\|e_{2}^{\varepsilon}\right\|_{H^{1}\left(P^{\varepsilon}\right)}+\left\|e_{2}^{\varepsilon}(p)\right\|_{H^{1}\left(P^{\varepsilon}\right)} \\
& +\varepsilon^{5 / 2}\left\|\underset{\sim}{\nabla} u^{2}-\underset{\sim}{\nabla} u^{2}(p)\right\|_{L^{2}(P)}+\varepsilon^{2}\left\|\partial_{\rho} Z\right\|_{L^{2}\left(P^{\varepsilon}\right)} .
\end{aligned}
$$

From Theorems 2.1 and 3.2, we have that $\left\|e_{2}^{\varepsilon}\right\|_{H^{1}\left(P^{\varepsilon}\right)}+\left\|e_{2}^{\varepsilon}(p)\right\|_{H^{1}(P)} \leq C \varepsilon^{3}$. The estimate for $\left\|\partial_{\rho} Z\right\|_{L^{2}\left(P^{\varepsilon}\right)}$ comes from Lemma 4.7. Finally we apply Lemma 4.6 to bound $\left\|\underset{\sim}{\nabla} u^{2}-\underset{\sim}{\nabla} u^{2}(p)\right\|_{L^{2}(P)}$, and substituting in (4.46) we have the result. The other estimates follow from similar arguments $\square$.

Remark 4.2 In the worst case scenario, when $g$ does not vanish identically along the boundary of $\partial P_{ \pm}$, i.e., $\sup _{\sim} \in \partial \Omega \max _{x_{3} \in\{-1,1\}}\left|g\left(\underset{\sim}{x}, x_{3}\right)\right| \neq 0$, then $\bar{\mu}\left(s^{*}, \delta\right)=$ $2-\delta$.

We summarize the convergence results in the table below. We present only the leading terms of the errors and in parenthesis we show interior estimates if those are better than the global ones. 
Table 2: Rates of convergence of the model error.

\begin{tabular}{c|c|c}
\hline norm & $u^{\varepsilon}-u^{\varepsilon}(p)$ & Relative Error \\
\hline$\|\cdot\|_{L^{2}\left(P^{\varepsilon}\right)}$ & $\varepsilon^{5 / 2} p^{-2-s} a_{s}$ & $\nu^{-2} \varepsilon^{2} p^{-2-s} a_{s}$ \\
$\left\|\partial_{\rho} \cdot\right\|_{L^{2}\left(P^{\varepsilon}\right)}$ & $\varepsilon^{2} p^{-\bar{\mu}} a_{s}^{b}\left(\varepsilon^{5 / 2} p^{-2-s} a_{s}^{1}\right)$ & $\nu^{-3 / 2} \varepsilon^{3 / 2} p^{-\bar{\mu}} a_{s}^{b}\left(\nu^{-2} \varepsilon^{2} p^{-2-s} a_{s}^{1}\right)$ \\
$\left\|\partial_{\theta} \cdot\right\|_{L^{2}\left(P^{\varepsilon}\right)}$ & $\varepsilon^{5 / 2} p^{-2-s} a_{s}^{1}$ & $\nu^{-2} \varepsilon^{2} p^{-2-s} a_{s}^{1}$ \\
$\left\|\partial_{x_{3}^{\varepsilon}} \cdot\right\|_{L^{2}\left(P^{\varepsilon}\right)}$ & $\varepsilon^{3 / 2} p^{-1-s} a_{s}$ & $p^{-1-s} a_{s}$ \\
$\|\cdot\|_{H^{1}\left(P^{\varepsilon}\right)}$ & $\varepsilon^{3 / 2} p^{-1-s} a_{s}$ & $\nu^{-1} \varepsilon p^{-1-s} a_{s}$ \\
\hline
\end{tabular}

The estimates of the table above indicate that the rate of convergence in $\varepsilon$ is the same regardless of the value of $p$. Nonetheless, increasing $p$ does diminish the modeling error, as expected. It is interesting to see that for the relative error norm, when $\zeta^{0} \equiv 0$ there is no convergence in $\varepsilon$, only in $p$. Finally, if $f$ is polynomial in the transverse direction, then $u^{2}=u^{2}(p)$ in this case, for $p$ high enough, and it is possible to obtain better convergence rates with respect to $\varepsilon$ in all norms of Table 2, with the exception of the $L^{2}\left(P^{\varepsilon}\right)$ norm of the normal derivative of the error. ${ }^{15}$

\section{Acknowledgment}

The first author was supported by NSF grant DMS-0107233. The second author had support from CNPq-Brazil.

\section{Appendix A}

In this appendix, we discuss several issues related to the boundary correctors. Our first goal is to prove existence, uniqueness and regularity of solutions for Poisson problems in the semi-infinite strip $\Sigma$. We also prove that under certain conditions, such solutions and their approximations decay exponentially. Next, we will study the properties of a standard Galerkin approximations for the boundary corrector $U^{2}$ in spaces with polynomial dependence in the vertical direction. We show stability and convergence results. We do not use the technique of separation of variables, although it would simplify some of the proofs, because it does not generalize to the case of linear elasticity.

In this appendix, we denote a typical point in $\Sigma$ by $\underset{\sim}{x}=\left(x_{1}, x_{2}\right)$. It is useful to consider the sets

$$
\Sigma(t, s)=\left\{\underset{\sim}{x} \in \Sigma: t<x_{1}<s\right\}, \quad \text { and } \quad \gamma_{t}=\left\{\underset{\sim}{x} \in \Sigma: x_{1}=t\right\},
$$

for $0 \leq t \leq s<\infty$. Let $V(\Sigma)=\left\{v \in \mathcal{D}^{\prime}(\Sigma): w v \in L^{2}(\Sigma), \underset{\sim}{\nabla} v \in \underset{\sim}{L^{2}}(\Sigma)\right\}$, where $w(\underset{\sim}{x})=\left(1+x_{1}\right)^{-1}$. By means of Hardy's inequality, it is possible to show that $V(\Sigma)$ endowed with the inner product $\int_{\Sigma} \underset{\sim}{\nabla} u \cdot \underset{\sim}{\nabla} v d \underset{\sim}{x}+\int_{\gamma_{0}} u v d x_{2}$ is a Hilbert space. ${ }^{15}$ 
We denote $V_{0}(\Sigma)$ as the set of functions in $V(\Sigma)$ that vanish on $\gamma_{0}$. The following well-posedness result holds.

Theorem A.1 Assume that $w^{-\alpha} f \in L^{2}(\Sigma)$, where $\alpha \geq 1$, and let $U_{0} \in H^{1 / 2}\left(\gamma_{0}\right)$. Then there exists unique $U \in V(\Sigma)$ such that

$$
\begin{gathered}
\int_{\Sigma} \underset{\sim}{\nabla} U \cdot \underset{\sim}{\nabla} v d \underset{\sim}{x}=\int_{\Sigma} f v d \underset{\sim}{\sim} \quad \text { for all } v \in V_{0}(\Sigma), \\
U=U_{0} \quad \text { on } \gamma_{0} .
\end{gathered}
$$

Moreover, there exists a constant $c$ independent of $f$ such that

$$
|U|_{H^{1}(\Sigma)} \leq \frac{2}{2 \alpha-1}\left\|w^{-\alpha} f\right\|_{L^{2}(\Sigma)}+c\left\|U_{0}\right\|_{H^{1 / 2}\left(\gamma_{0}\right)} .
$$

With the questions of existence and uniqueness answered, we proceed to further characterize the boundary correctors. We show that they decay exponentially fast to a constant, in a sense that we will make clear. Our proof generalizes previous approaches. ${ }^{12}$ It allows a nontrivial right hand side, and, more importantly, it works not only for the exact solution of (A.1), but also for some of its approximations. So, below, $\bar{U}$ does not necessarily solves (A.1), but it might be the projection of the solution into some particular space. Similarly, $\underset{\sim}{\bar{\sigma}}$ might be either the gradient of the solution or its approximation. In our applications, $\bar{U}$ and $\underset{\sim}{\bar{\sim}}$ are given by Galerkin or mixed approximations. As we see below, sufficient conditions for such exponential decay are that $\bar{U} \in L_{w}^{2}(\Sigma), \underset{\sim}{\bar{\sigma}} \in \underset{\sim}{L^{2}}(\Sigma)$, and that $\bar{U}, \underset{\sim}{\bar{\sigma}}$ satisfy for $0 \leq t \leq s<\infty$ :

$$
\begin{aligned}
& \int_{\Sigma(t, s)} \mid \underset{\sim}{|\bar{\sigma}|^{2}} d \underset{\sim}{x}=\int_{\Sigma(t, s)} f \bar{U} d \underset{\sim}{\sim}-\int_{\gamma_{t}} \bar{\sigma}_{1} \bar{U} d x_{2}+\int_{\gamma_{s}} \bar{\sigma}_{1} \bar{U} d x_{2}, \\
& \int_{\Sigma(t, s)} f d \underset{\sim}{\sim}=\int_{\gamma_{t}} \bar{\sigma}_{1} d x_{2}-\int_{\gamma_{s}} \bar{\sigma}_{1} d x_{2} \\
& -\int_{\Sigma(0, t)} x_{1} f d x=\int_{\gamma_{0}} \bar{U} d x_{2}+\int_{\gamma_{t}}\left(t \bar{\sigma}_{1}-\bar{U}\right) d x_{2} \\
& \int_{\gamma_{t}} \bar{U}^{2} d x_{2} \leq c_{W} \int_{\gamma_{t}} \bar{\sigma}_{2}^{2} d x_{2}+\frac{1}{2}\left(\int_{\gamma_{t}} \bar{U} d x_{2}\right)^{2} \quad \text { for some } c_{W} \geq 0 \text {. }
\end{aligned}
$$

The constant $c_{W}$ in the condition (C4) mimics the Wirtinger inequality (the onedimensional version of the Poincaré's inequality $\left.{ }^{18}\right)$.

Assume that there exist positive constants $c_{0}$ and $M$ such that

$$
\left(\int_{\Sigma(t, \infty)} f(\underset{\sim}{x})^{2} d \underset{\sim}{x}\right)^{1 / 2}+\left|\int_{\Sigma(t, \infty)}\left(t-x_{1}\right) f(\underset{\sim}{x}) d \underset{\sim}{x}\right| \leq M \exp \left(-c_{0} t\right)
$$

and define

$$
\left.c_{\infty}(\bar{U})=\int_{\Sigma} x_{1} f \underset{\sim}{x}\right) d \underset{\sim}{x}+\int_{\gamma_{0}} \bar{U} d x_{2} .
$$

In the following two lemmas we show that results similar to $(\mathrm{C} 1)-(\mathrm{C} 3)$ are valid in unbounded sections of $\Sigma$ as well. 
Lemma A.1 Assume that (A.3) holds, $w U \in L^{2}(\Sigma), \underset{\sim}{\sigma} \in \underset{\sim}{L^{2}}(\Sigma)$ and that conditions (C2), (C3) are satisfied. Then for $t \geq 0$

$$
\begin{gathered}
\int_{\Sigma(t, \infty)} f d \underset{\sim}{x}=\int_{\gamma_{t}} \sigma_{1} d x_{2} \\
\int_{\gamma_{t}} U d x_{2}=c_{\infty}(U)+\int_{\Sigma(t, \infty)}\left(t-x_{1}\right) f(\underset{\sim}{x}) d \underset{\sim}{x} .
\end{gathered}
$$

Proof. If we define $P(s)=\int_{\gamma_{s}} \sigma_{1} d x_{2}$, then in view of $(\mathrm{C} 2)$ we have that

$$
P(s)=\int_{\gamma_{t}} \sigma_{1} d x_{2}-\int_{\Sigma(t, s)} f d \underset{\sim}{x}
$$

Thus $P$ is a continuous function and $\lim _{s \rightarrow \infty} P(s)=d$, where $d$ is the constant

$$
d=\int_{\gamma_{t}} \sigma_{1} d x_{2}-\int_{\Sigma(t, \infty)} f d x .
$$

Since $|\underset{\sim}{\sigma}| \in L^{2}(\Sigma)$, then $P(s) \in L^{2}\left(\mathbb{R}^{+}\right)$. Hence $d=0$ and identity (A.5) follows. Now, to conclude (A.6), we use (C3) and then Eqs. (A.4), (A.5) $\square$.

The proof of the lemma below follows from similar arguments

Lemma A.2 Assume that $U,|\underset{\sim}{\sigma}| \in L^{2}(\Sigma)$ and that condition $(C 1)$ is satisfied. Then, for $t \geq 0$

$$
\int_{\Sigma(t, \infty)}|\underset{\sim}{\mid \sigma}|^{2} d \underset{\sim}{x}=-\int_{\gamma_{t}} \sigma_{1} U d x_{2}+\int_{\Sigma(t, \infty)} f U d \underset{\sim}{x}
$$

We have the following results.

Theorem A.2 Assume that (A.3) holds, that $w \bar{U} \in L^{2}(\Sigma), \underset{\sim}{\bar{\sigma}} \in \underset{\sim}{L^{2}}(\Sigma)$ satisfy (C1)-(C4), and also that $c_{\infty}(\bar{U})=0$. Then there exists a constant $c$ depending only on $c_{0}$ and $c_{W}$ such that

$$
\int_{\Sigma(t, \infty)} \bar{U}^{2}+|\underset{\sim}{\bar{\sigma}}|^{2} d \underset{\sim}{x} \leq c\left(1+\int_{\Sigma}|\bar{\sigma}|^{2} d \underset{\sim}{x}\right) \exp \left(-t / c_{1}\right)
$$

where $c_{1}=\max \left\{1+c_{W}, 1 / c_{0}\right\}$.

Proof. Let $I(t)=\int_{\gamma_{t}} \bar{U} d x_{2}$. Then, from Lemma A.1 and Eq. (A.3),

$$
|I(t)| \leq M \exp \left(-c_{0} t\right)
$$

If we define the function $E(t)=\int_{\Sigma(t, \infty)}|\underset{\sim}{\sigma}|^{2} d \underset{\sim}{x}$, then $E^{\prime}(t)=-\int_{\gamma_{t}}|\bar{\sim}|^{2} d x_{2}$ and (C4) yields

$$
\begin{gathered}
\int_{\gamma_{t}} \bar{U}^{2} d x_{2} \leq-c_{W} E^{\prime}(t)+\frac{I(t)^{2}}{2} \\
\int_{\Sigma(t, \infty)} \bar{U}^{2} d x \leq \int_{t}^{\infty}\left(c_{W} \int_{\gamma_{x_{1}}}|\bar{\sim}|^{2} d x_{2}+\frac{1}{2} I\left(x_{1}\right)^{2}\right) d x_{1} \\
=c_{W} E(t)+\frac{1}{2} \int_{t}^{\infty} I\left(x_{1}\right)^{2} d x_{1} .
\end{gathered}
$$


We can now bound the growth of the energy. From (A.9) and (A.11), we conclude that $\bar{U} \in L^{2}(\Sigma)$, and using Lemma A.2 we gather that:

$$
\begin{aligned}
E(t)= & -\int_{\gamma_{t}} \bar{\sigma}_{1} \bar{U} d x_{2}+\int_{\Sigma(t, \infty)} f \bar{U} d \underset{\sim}{x} \\
\leq & \frac{1}{2} \int_{\gamma_{t}} \bar{\sigma}_{1}^{2} d x_{2}+\frac{1}{2} \int_{\gamma_{t}} \bar{U}^{2} d x_{2}+\frac{\alpha}{2} \int_{\Sigma(t, \infty)} \bar{U}^{2} d \underset{\sim}{x}+\frac{1}{2 \alpha} \int_{\Sigma(t, \infty)} f^{2} d \underset{\sim}{\alpha} \\
\leq & -\frac{\left(1+c_{W}\right)}{2} E^{\prime}(t)+\frac{I(t)^{2}}{4}+\frac{\alpha c_{W}}{2} E(t)+\frac{\alpha}{4} \int_{t}^{\infty} I\left(x_{1}\right)^{2} d x_{1} \\
& +\frac{1}{2 \alpha} \int_{\Sigma(t, \infty)} f^{2} d \underset{\sim}{x},
\end{aligned}
$$

where (A.10) and (A.11) were used in the last inequality. Choose $\alpha=\left(c_{W}\right)^{-1}$ in (A.12) to conclude that (recall that $E^{\prime}(t)$ is nonpositive):

$$
c_{1} E^{\prime}(t) \leq\left(1+c_{W}\right) E^{\prime}(t) \leq-E(t)+G(t),
$$

where

$$
c_{1}=\max \left\{1+c_{W}, \frac{1}{c_{0}}\right\}, \quad G(t)=\frac{I(t)^{2}}{2}+\frac{1}{2 c_{W}} \int_{t}^{\infty} I\left(x_{1}\right)^{2} d x_{1}+c_{W} \int_{\Sigma(t, \infty)} f^{2} d \underset{\sim}{\sim} .
$$

We estimate now the energy norm. Define $W(t)$ such that

$$
W^{\prime}(t)=-\frac{W(t)}{c_{1}}+\frac{G(t)}{c_{1}}, \quad W(0)=E(0) .
$$

Then

$$
E(t) \leq W(t)=\frac{1}{c_{1}} \exp \left(-t / c_{1}\right) \int_{0}^{t} \exp \left(x_{1} / c_{1}\right) G\left(x_{1}\right) d x_{1}+E(0) \exp \left(-t / c_{1}\right) .
$$

Using (A.14), (A.3), and (A.9) we have that the integral in (A.15) is uniformly bounded and then $E(t)$ decays exponentially. Combining (A.9) and (A.11), we have the corresponding decay of $\|\bar{U}\|_{L^{2}(\Sigma(t, \infty))} \square$.

Using the previous theorem, we can decompose a general solution as a constant term plus a exponentially decaying function, as the result below shows.

Corollary A.1 Assume that (A.3) holds and that $\bar{U} \in V(\Sigma), \underset{\sim}{\bar{\sigma}} \in L^{2}(\Sigma)$ satisfy (C1)-(C4). Defining $c_{\infty}(\bar{U})$ as in (A.4), we have the decomposition

$$
\bar{U}=\frac{1}{2} c_{\infty}(\bar{U})+\bar{U}^{*}
$$

where $\bar{U}^{*}, \underset{\sim}{\bar{\sigma}}$ decay to zero exponentially as in Theorem A.2, i.e., (A.8) is satisfied with $\bar{U}$ replaced by $\bar{U}^{*}$. 
In the rest of this appendix, we investigate how well elements of $V(\Sigma, p)$ can approximate the solution of

$$
\begin{gathered}
\Delta U=0 \quad \text { in } \Sigma, \\
\frac{\partial U}{\partial n}=0 \quad \text { on } \mathbb{R}^{+} \times\{-1,1\}, \\
U=U_{0} \quad \text { on } \gamma_{0},
\end{gathered}
$$

where $U_{0} \in H^{r_{0}}\left(\gamma_{0}\right) \cap \hat{L}^{2}\left(\gamma_{0}\right)$ for some $r_{0}>3 / 2$. The approximation rates are severely limited by the presence of corner singularities in $U$. We describe these singularities explicitly and expose their influence in the convergence rates.

To describe the singular behavior of the solution of (A.17), we introduce in $\Sigma$ two polar coordinate systems, $\left(r_{l}, \theta_{l}\right), l=1,2$ relative to the vertices $P_{1}=(0,1)$ and $P_{2}=(0,-1)$. The convention is that $r_{l}$ gives the distance to $P_{l}$ and the angle $\theta_{l} \in[0, \pi / 2]$ increases counterclockwise, so points lying on $\gamma_{0}$ have $\theta_{1}=0$ and $\theta_{2}=\pi / 2$.

The next theorem, ${ }^{13}$ shows a decomposition of the solution $U$ in singular and smooth parts and it is of paramount importance in future estimates.

Theorem A.3 Let $U \in V(\Sigma)$ be the solution of (A.17) with $r_{0}>3 / 2$ such that $r_{0}+1 / 2$ is not an even integer. Then there exist constants $c_{j}$ such that

$$
U=U_{S}+W, \quad U_{S}=\check{\chi} \sum_{l=1}^{2} \sum_{j=1}^{N\left(r_{0}+1 / 2\right)} c_{j} \partial_{2}^{(2 j-1)} U_{0}\left((-1)^{l+1}\right) v_{l}^{j},
$$

where $\check{\chi}$ is a smooth cutoff function that equals the identity for $x_{1}<1$ and vanishes for $x_{1}>2, N$ is as in (4.44), and

$$
\begin{gathered}
v_{1}^{j}=\left[\theta_{1} \cos \left((2 j-1) \theta_{1}\right)+\log r_{1} \sin \left((2 j-1) \theta_{1}\right)\right] r_{1}^{(2 j-1)}, \\
v_{2}^{j}=\left[\left(\frac{\pi}{2}-\theta_{2}\right) \sin \left((2 j-1) \theta_{2}\right)+\log r_{2} \cos \left((2 j-1) \theta_{2}\right)\right] r_{2}^{(2 j-1)} .
\end{gathered}
$$

Furthermore, $\|W\|_{H^{r_{0}+1 / 2}(\Sigma)} \leq c\left\|U_{0}\right\|_{H^{r_{0}\left(\gamma_{0}\right)}}$ for some constant $c$.

Remark A.1 Note that $v_{1}^{j}=v_{2}^{j}=0$ when $x_{1}=0$, and therefore $U_{S}$ is identically zero at $x_{1}=0$.

Remark A.2 Since $U_{0} \in H^{r_{0}}(-1,1)$ and $2 N\left(r_{0}+1 / 2\right)-1<r_{0}-1 / 2$, then $U_{S}$ is well defined. Also, note that the singular behavior of $U$ depends not only on the regularity of the Dirichlet data $U_{0}$ but also on how many derivatives of $U_{0}$ vanish at the endpoints $-1,1$. For instance, although $U_{0}(y)=y$ is smooth, it gives rise to a singular solution.

Let $\hat{\pi}_{p}^{1\left(x_{2}\right)}$ be the operator that acts like $\hat{\pi}_{p}^{1}$ in each vertical fiber, i.e., if $\phi \in$ $L^{2}\left(\mathbb{R}^{+} ; H^{1}(-1,1) \cap \hat{L}^{2}(-1,1)\right)$, then $\hat{\pi}_{p}^{1\left(x_{2}\right)} \phi \in L^{2}\left(\mathbb{R}^{+} ; \hat{\mathbb{P}}_{p}(-1,1)\right)$, and

$$
\int_{\Sigma} \partial_{2}\left(\phi-\hat{\pi}_{p}^{1\left(x_{2}\right)} \phi\right) \partial_{2} \psi d x=0 \quad \text { for all } \psi \in L^{2}\left((-1,1) ; \hat{\mathbb{P}}_{p}(-1,1)\right) .
$$


Furthermore, let $\pi_{p}$ be the orthogonal $L^{2}$ projection operator from $L^{2}(\Sigma)$ into $L^{2}\left(\mathbb{R}^{+} ; \mathbb{P}_{p}(-1,1)\right)$, and let $\Pi_{p}^{1}$ be the orthogonal $H^{1}$ projection operator from $H^{1}(\Sigma)$ into $H^{1}\left(\mathbb{R}^{+} ; \mathbb{P}_{p}(-1,1)\right)$.

To estimate the projection error of the singular function $U_{S}$, we apply the ideas of Dorr, ${ }^{10,11}$ and the Remark 6.3 of Bernardi and Maday. ${ }^{5}$ See the Madureira's thesis ${ }^{15}$ for a description of how such convergence rates can be obtained.

Lemma A.3 Let $v(r, \theta)=\check{\chi}^{\alpha}\left[\xi_{1}(\theta)+\xi_{2}(\theta) \log r\right]$, where $\xi_{1}, \xi_{2} \in C^{\infty}([0, \pi / 2])$, and $\alpha$ is a nonnegative real number. Then, for every $\delta$, there exists a constant $c$ such that

$$
\left\|v-\pi_{p}^{\left(\tilde{\rho}_{2}\right)} v\right\|_{H^{1}(\Sigma)} \leq c p^{-2 \alpha+\delta} .
$$

The result below estimates approximations given by projection operators, based on the decomposition (A.18).

Lemma A.4 Assume that $U \in V(\Sigma)$ solves (A.17) with $r_{0}>3 / 2$ such that $r_{0}+1 / 2$ is not an even integer, and that $W$ and $U_{S}$ are as in (A.18). Then, there exists a constant $c$ such that

$$
\left\|W-\pi_{p}^{1\left(x_{2}\right)} W\right\|_{H^{1}(\Sigma)} \leq c p^{1 / 2-r_{0}}\left\|U_{0}\right\|_{H^{r_{0}}\left(\gamma_{0}\right)} .
$$

Also, if $U_{S}$ is not the zero function then for any arbitrarily small $\delta>0$, there exists a constant $c$ such that

$$
\left\|U_{S}-\pi_{p}^{\left(x_{2}\right)} U_{S}\right\|_{H^{1}(\Sigma)} \leq c p^{-4 m+2+\delta}\left\|U_{0}\right\|_{H^{r_{0}}\left(\gamma_{0}\right)},
$$

where $m \in\left\{1, \ldots, N\left(r_{0}+\frac{1}{2}\right)\right\}$ is the minimum integer such that

$$
\left|\partial_{2}^{(2 m-1)} U_{0}(-1)\right|+\left|\partial_{2}^{(2 m-1)} U_{0}(1)\right| \neq 0 .
$$

Remark A.3 Using the work of Babuška and Suri, ${ }^{2}$ it is possible to improve the estimate of Lemma A.4 slightly, replacing $p^{-4 m+2+\delta}$ by $p^{-4 m+2}(\log p)$, at the expense of many technicalities.

We define the rate of convergence of our approximation result below.

Definition A.1 For $U_{0} \in H^{r_{0}}(-1,1)$, and $N$ as in (4.44), if there exists an minimum integer $m \in\left\{1, \ldots, N\left(r_{0}+\frac{1}{2}\right)\right\}$ such that $\left|\partial_{2}^{2 m-1} U_{0}(-1)\right|+\left|\partial_{2}^{2 m-1} U_{0}(1)\right| \neq 0$, let $\gamma\left(r_{0}, \delta\right)=\min \left\{4 m-2-\delta, r_{0}-1 / 2\right\}$, otherwise let $\gamma\left(r_{0}, \delta\right)=r_{0}-1 / 2$.

We conclude now the following approximation result for $U$.

Theorem A.4 Assume that $U$ solves (A.17) with $r_{0}>3 / 2$ such that $r_{0}+1 / 2$ is not an even integer. Then

$$
\left\|U-\Pi_{p}^{1} U\right\|_{H^{1}(\Sigma)} \leq c p^{-\gamma\left(r_{0}, \delta\right)}\left\|U_{0}\right\|_{H^{r_{0}}\left(\gamma_{0}\right)},
$$

where $\gamma$ is as in Definition A.1. The constant c depends on $r_{0}$ and $\delta>0$ only.

Proof. Using the best approximation property of $\Pi_{p}^{1}$, Theorem A.3 and Lemma A.4, we have that

$$
\begin{aligned}
\left\|U-\Pi_{p}^{1} U\right\|_{H^{1}(\Sigma)} \leq\left\|U_{S}-\pi_{p}^{\left(x_{2}\right)} U_{S}\right\|_{H^{1}(\Sigma)}+\left\|W-\pi_{p}^{1\left(x_{2}\right)} W\right\|_{H^{1}(\Sigma)} \\
\leq c p^{-\gamma\left(r_{0}, \delta\right)}\left\|U_{0}\right\|_{H^{r_{0}}\left(\gamma_{0}\right)}
\end{aligned}
$$


$\square$.

Now we use the above result to estimate the errors due to the Galerkin projections.

Theorem A.5 For any real number $r_{0}>3 / 2$ such that $r_{0}+1 / 2$ is not an even integer, and any arbitrarily small $\delta>0$, there exists a constant $c$ such that if $U \in V(\Sigma)$ solves (A.17) with $U_{0} \in H^{r_{0}}\left(\gamma_{0}\right) \cap \hat{L}^{2}\left(\gamma_{0}\right)$, and if $U(p) \in V(\Sigma, p)$ solves

$$
\begin{gathered}
\int_{\Sigma} \underset{\sim}{\nabla} U(p) \cdot \underset{\sim}{\nabla} v d \underset{\sim}{\sim}=0 \quad \text { for all } v \in V_{0}(\Sigma, p), \\
U(p)=\hat{\pi}_{p}^{1} U_{0} \quad \text { on } \gamma_{0},
\end{gathered}
$$

then

$$
|U-U(p)|_{H^{1}(\Sigma)} \leq c p^{-\gamma\left(r_{0}, \delta\right)}\left\|U_{0}\right\|_{H^{r_{0}}\left(\gamma_{0}\right)},
$$

where $\gamma$ is as in Definition A.1.

Proof. Let $\tilde{U}_{0}$ be the trace of $\Pi_{p}^{1} U$ on $\gamma_{0}$. Then, from the trace Theorem and Theorem A.4,

$$
\left\|U_{0}-\tilde{U}_{0}\right\|_{H^{1 / 2}\left(\gamma_{0}\right)} \leq c\left\|U-\Pi_{p}^{1} U\right\|_{H^{1}(\Sigma)} \leq c p^{-\gamma\left(r_{0}, \delta\right)}\left\|U_{0}\right\|_{H^{r_{0}}\left(\gamma_{0}\right)} .
$$

Also,

$$
\begin{aligned}
\left\|\tilde{U}_{0}-\hat{\pi}_{p}^{1} U_{0}\right\|_{H^{1 / 2}\left(\gamma_{0}\right)} \leq\left\|\tilde{U}_{0}-U_{0}\right\|_{H^{1 / 2}\left(\gamma_{0}\right)}+\left\|U_{0}-\hat{\pi}_{p}^{1} U_{0}\right\|_{H^{1 / 2}\left(\gamma_{0}\right)} \\
\leq c p^{-\gamma\left(r_{0}, \delta\right)}\left\|U_{0}\right\|_{H^{r_{0}}\left(\gamma_{0}\right)} .
\end{aligned}
$$

Introduce now $\tilde{U} \in V(\Sigma)$ such that

$$
\begin{array}{cl}
\int_{\Sigma} \underset{\sim}{\nabla} \tilde{U} \cdot \underset{\sim}{\nabla} v d \underset{\sim}{x}=0 & \text { for all } v \in V_{0}(\Sigma), \\
\tilde{U}=\tilde{U}_{0} & \text { on } \gamma_{0},
\end{array}
$$

and also $\tilde{U}(p) \in V(\Sigma, p)$ such that

$$
\begin{array}{cl}
\int_{\Sigma} \underset{\sim}{\nabla} \tilde{U}(p) \cdot \underset{\sim}{\nabla} v d \underset{\sim}{x}=0 & \text { for all } v \in V_{0}(\Sigma, p), \\
\tilde{U}(p)=\tilde{U}_{0} & \text { on } \gamma_{0},
\end{array}
$$

Then,

$$
\begin{aligned}
& |U-\tilde{U}|_{H^{1}(\Sigma)}+|\tilde{U}(p)-U(p)|_{H^{1}(\Sigma)} \\
& \quad \leq c\left\|U_{0}-\tilde{U}_{0}\right\|_{H^{1 / 2}\left(\gamma_{0}\right)}+c\left\|\tilde{U}_{0}-\hat{\pi}_{p}^{1} U_{0}\right\|_{H^{1 / 2}\left(\gamma_{0}\right)} \leq c p^{-\gamma\left(r_{0}, \delta\right)}\left\|U_{0}\right\|_{H^{r_{0}}\left(\gamma_{0}\right)} .
\end{aligned}
$$

Now we advance to estimate $|\tilde{U}-\tilde{U}(p)|_{H^{1}(\Sigma)}$. Since $\tilde{U}(p)-\Pi_{p}^{1} U \in V_{0}(\Sigma, p)$, then

$$
\begin{aligned}
\left|\tilde{U}(p)-\Pi_{p}^{1} U\right|_{H^{1}(\Sigma)}^{2}=\int_{\Sigma} \underset{\sim}{\nabla}\left(\tilde{U}(p)-\Pi_{p}^{1} U\right) & \cdot \underset{\sim}{\nabla}\left(\tilde{U}-\Pi_{p}^{1} U\right) d \underset{\sim}{x} \\
& \leq\left|\tilde{U}(p)-\Pi_{p}^{1} U\right|_{H^{1}(\Sigma)}\left|\tilde{U}-\Pi_{p}^{1} U\right|_{H^{1}(\Sigma)},
\end{aligned}
$$


and therefore, $\left|\tilde{U}(p)-\Pi_{p}^{1} U\right|_{H^{1}(\Sigma)} \leq\left|\tilde{U}-\Pi_{p}^{1} U\right|_{H^{1}(\Sigma)}$. So, using the triangle inequality

$$
\begin{aligned}
|\tilde{U}-\tilde{U}(p)|_{H^{1}(\Sigma)} & \leq\left|\tilde{U}-\Pi_{p}^{1} U\right|_{H^{1}(\Sigma)}+\left|\Pi_{p}^{1} U-\tilde{U}(p)\right|_{H^{1}(\Sigma)} \leq 2\left|\tilde{U}-\Pi_{p}^{1} U\right|_{H^{1}(\Sigma)} \\
& \leq 2|\tilde{U}-U|_{H^{1}(\Sigma)}+2\left|U-\Pi_{p}^{1} U\right|_{H^{1}(\Sigma)} \leq c p^{-\gamma\left(r_{0}, \delta\right)}\left\|U_{0}\right\|_{H^{r_{0}}\left(\gamma_{0}\right)}
\end{aligned}
$$

from (A.19) and from Theorem A.4. Finally,

$$
\begin{aligned}
|U-U(p)|_{H^{1}(\Sigma)} \leq|U-\tilde{U}|_{H^{1}(\Sigma)}+|\tilde{U}-\tilde{U}(p)|_{H^{1}(\Sigma)}+ & |\tilde{U}(p)-U(p)|_{H^{1}(\Sigma)} \\
& \leq c p^{-\gamma\left(r_{0}, \delta\right)}\left\|U_{0}\right\|_{H^{r_{0}}\left(\gamma_{0}\right)},
\end{aligned}
$$

and the result follows $\square$.

Remark A.4 It is interesting to see how the corner singularities spoil an otherwise good convergence rate. For example, if $U_{0}(y)=y$, the Galerkin projection converges as $p^{-2+\delta}$ in $H^{1}(\Sigma)$, while if $U_{0}$ is still smooth but has all derivatives vanishing at the endpoints, then the convergence is faster than polynomial. ${ }^{15}$

\section{Appendix B}

In this second part of the appendix, we include proofs of results stated throughout the paper and which proofs use results developed in the first part of this appendix.

Proof. (of Lemma 2.2) We use induction on $k$ to prove the result and the relation

$$
\int_{\Sigma} \tilde{\rho}^{l} \partial_{\tilde{\rho}} U^{k} d \tilde{\rho} d x_{3}=\int_{\Sigma} \tilde{\rho}^{l} U^{k} d \tilde{\rho} d x_{3}=0, \quad l=0,1, \cdots
$$

Recall that, by convention, $U^{1}=0$, and assume that the lemma and (B.1) hold for $k=1, \cdots, K-1$. We show now that the same holds for $k=K$. From the definition of $F_{K}$, the hypotheses of Theorem A.1 are fulfilled, and there exists a unique function $U^{K}$ solving (2.22) such that $\left|\partial_{\tilde{\rho}} U^{k}\right|+\left|\partial_{3} U^{k}\right| \in L^{2}(\Sigma)$. To conclude (2.24), we first note from Corollary A.1 that $U^{K}$ decays toward the constant $c_{K}(\theta)=\int_{\Sigma} \tilde{\rho} F_{K}\left(\tilde{\rho}, \theta, x_{3}\right) d \tilde{\rho} d x_{3}+\int_{-1}^{1} u^{K}\left(0, \theta, x_{3}\right) d x_{3}$. Our goal now is to show that this constant is actually zero. Since $u^{K}$ has zero average in each fiber, using the definition of $F_{k}$, it is enough to prove (B.1) for any positive integer $l$. Using the formula

$$
\int_{\Sigma} u \Delta v d \tilde{\rho} d x_{3}=\int_{\Sigma} v \Delta u d \tilde{\rho} d x_{3}+\int_{\partial \Sigma} u \frac{\partial v}{\partial n}-v \frac{\partial u}{\partial n} d \tilde{\rho} d x_{3},
$$

with $u=U^{K}$ and $v=\tilde{\rho}^{l+2} /[(l+2)(l+1)]$, we get

$$
\int_{\Sigma} \tilde{\rho}^{l} U^{K} d \tilde{\rho} d x_{3}=\int_{\Sigma} \frac{\tilde{\rho}^{l+2}}{(l+2)(l+1)} F_{K} d \tilde{\rho} d x_{3}=0
$$

from the definition of $F_{K}$ and the inductive hypothesis. Similarly, using integration by parts, we also have that $\int_{\Sigma} \tilde{\rho}^{l} \partial_{\tilde{\rho}} U^{K} d \tilde{\rho} d x_{3}=0$. Hence (B.1) holds and the lemma 
follows $\square$.

Proof. (of Lemma 4.7) From Theorem A.5, with $u^{2}(0, \theta, \cdot)$ replacing $U_{0}$, we have that for each $\theta$,

$$
\left|U^{2}-U^{2}(p)\right|_{H^{1}(\Sigma)} \leq c p^{-\gamma(s+2, \delta)}\left\|u^{2}(0, \theta, \cdot)\right\|_{H^{s+2}(-1,1)} .
$$

Changing coordinates, we have that

$$
\begin{aligned}
& \left\|\partial_{\rho} Z\right\|_{L^{2}\left(P^{\varepsilon}\right)}^{2} \\
& \leq c \int_{0}^{L} \int_{\Sigma}\left|\partial_{\rho}\left[U^{2}-U^{2}(p)\right]\right|^{2} d \tilde{\rho} d x_{3} d \theta+c \int_{0}^{L} \int_{\Sigma}\left|\chi^{\prime}\right|^{2}\left[U^{2}-U^{2}(p)\right]^{2} d \tilde{\rho} d x_{3} d \theta \\
& \quad \leq c p^{-\gamma(s+2, \delta)}\left\|u^{2}(0, \theta, \cdot)\right\|_{H^{s+2}(-1,1)},
\end{aligned}
$$

where we used Lemma 2.3 and Eqs. (3.42) and (B.2) in the last inequality. Now, from the definitions 4.3 and A.1, $\gamma(r+2, \delta)=\bar{\mu}(s, \delta)$, and from Lemma 2.1, we have that

$$
\left\|u^{2}(0, \theta, \cdot)\right\|_{H^{r+2}(-1,1)} \leq a_{s}^{b},
$$

and the result follows $\square$.

Proof. (of Lemma 4.7) For $\underset{\sim}{x} \in \partial \Omega$ fixed, let $\theta$ be such that $\underset{\sim}{z}(\theta)=\underset{\sim}{x}$. From Eqs (2.22), (3.41), and Theorem A.5, there exists a constant $c$ such that

$$
\begin{array}{r}
\int_{\Sigma}\left|\partial_{\tilde{\rho}}\left[U^{2}-U^{2}(p)\right]\right|^{2}+\left|\partial_{x_{3}}\left[U^{2}-U^{2}(p)\right]\right|^{2} d \tilde{\rho} d x_{3} \leq c p^{-\gamma(s+2, p)}\left\|u^{2}(\underset{\sim}{x}, \cdot)\right\|_{H^{s+2}(-1,1)} \\
\leq c p^{-\gamma(s+2, p)}\left(\|f(\underset{\sim}{x}, \cdot)\|_{H^{s}(-1,1)}+|g(\underset{\sim}{x},-1)|+|g(\underset{\sim}{x}, 1)|\right) . \quad \text { (B.3) }
\end{array}
$$

Replacing $U_{0}(\cdot)$ by $u^{2}(\underset{\sim}{x}, \cdot)$ in Definition A.1, and using (2.9), (2.11), and (2.16), it is not hard to show that $\gamma(s+2, p) \leq \bar{\mu}(s, \delta)$. Integrating (B.3) in $\theta$ and using Lemma 2.1, we conclude the proof $\square$.

\section{References}

1. D. N. Arnold and R. S. Falk, Asymptotic analysis of the boundary layer for the ReissnerMindlin plate model, SIAM J. Math. Anal. 27 (1996) 468-514.

2. I. Babuška and M. Suri, The optimal convergence rate of the p-version of the finite element method, SIAM J. Numer. Anal. 24 (1987) 750-776.

3. I. Babuška and L. Li, Hierarchical modelling of plates, Computers and Structures $\mathbf{4 0}$ (1991) 419-430.

4. I. Babuška and L. Li, The problem of plate modeling: Theoretical and computational results, Comput. Methods Appl. Mech. Engrg. 100 (1992) 249-273.

5. C. Bernardi and Y. Maday, Spectral Methods, Handbook of Numerical Analysis, eds. P. G. Ciarlet and J. L. Lions Vol. V (Elsevier Science, 1997).

6. C. Chen, Asymptotic convergence rates for the Kirchhoff plate model, Ph.D. Dissertation, The Pennsylvania State University (1995). 
7. P. G. Ciarlet, Mathematical Elasticity, volume II: Theory of Plates, Studies in mathematics and its applications. Vol. 27 (Elsevier Science B. V., 1997).

8. M. Dauge and I. Gruais, Asymptotics of arbitrary order for a thin elastic clamped plate, I: Optimal error estimates, Asymptotic Analysis 13 (1996) 167-197.

9. M. Dauge, I. Gruais and A. Rössle, The influence of lateral boundary conditions on the asymptotics in thin elastic plates, SIAM J. Math. Anal. 31 (2000) 305-345.

10. M. Dorr, The approximation theory for the p-version of the finite element method, SIAM J. Numer. Anal. 21 (1984) 1180-1207.

11. M. Dorr, The approximation of solutions of elliptic boundary-value problems via the p-version of the finite element method, SIAM J. Numer. Anal. 23 (1986) 58-77.

12. C. O. Horgan and J. K. Knowles, Recent Developments Concerning Saint-Venant's Priciple, Advances in Applied Mechanics 23 (1983) 179-269.

13. R. B. Kellogg, Notes on piecewise smooth elliptic boundary value problems, Institute for Physical Science and Technology, Technical Note BN-1137 (1992).

14. K. H. Lo, R. M. Christensen and E. M. Wu, A high-Order Theory of Plate Deformation, J. Appl. Mech. 46 (1977) 663-676.

15. A. L. Madureira, Asymptotics and Hierarchical Modeling of Thin Plates, Ph.D. Dissertation, The Pennsylvania State University (1999).

16. W. G. Mazja and S. A. Nazarow and B. A. Plamenewski, Asymptotische Theorie Ellipscher Randwertaufgaben in Singulär gestörten Gebieten I (Akademie Verlag, 1991).

17. B. Miara, Optimal Spectral Approximation in Linearized Plate Theory, Applicable Analysis 31 (1989) 291-307.

18. D. S. Mitrinović and J. E. Pečarić and A. M. Fink, Inequalities Involving Functions and their Integrals and Derivatives (Kluwer Academic Publishers, 1991).

19. O. Ovaskainen, J. Pitkäranta, An energy method approach to the problem of elastic strip, SIAM J. Appl. Math. 58 (1998) 999-1021.

20. C. Schwab, Wavelets, Multilevel Methods and Elliptic PDEs, in Hierarchic modelling in mechanics, eds. M. Ainsworth, J. Levesley, W.A. Light and M. Marletta (Oxford University Press, 1997), pp. 85-160.

21. M. Vogelius and I. Babuška, On a dimensional reduction method I. The optimal Selection of basis functions, Mathematics of computation 37 (1981) 31-46.

22. M. Vogelius and I. Babuška, On a dimensional reduction method II. Some approximation-theoretic results, Mathematics of computation 37 (1981) 47-68.

23. M. Vogelius and I. Babuška, On a dimensional reduction method III. A posteriori error estimation and an adaptive approach, Mathematics of computation 37 (1981) 361-384. 